\title{
PARP1 Inhibitor and Trabectedin Combination Does Not Increase Tumor Mutational Burden in Advanced Sarcomas-A Preclinical and Translational Study
}

\author{
Ymera Pignochino ${ }^{1,2,+}$, Giovanni Crisafulli ${ }^{2,3,+} \mathbb{0}$, Giorgia Giordano ${ }^{2,3}$, Alessandra Merlini ${ }^{2,3, *}$, \\ Enrico Berrino $^{2,4}$, Maria Laura Centomo ${ }^{2,3}$, Giulia Chiabotto ${ }^{4}$, Silvia Brusco ${ }^{2,3}$, Marco Basiricò ${ }^{2}$,

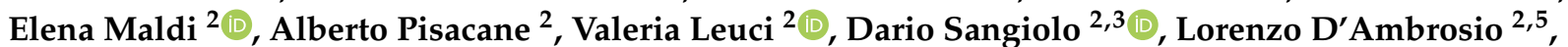 \\ Massimo Aglietta ${ }^{2,3}$, Bernd Kasper ${ }^{6}{ }^{-}$, Alberto Bardelli ${ }^{2,3}$ and Giovanni Grignani ${ }^{2} \mathbb{}$
}

1 Department of Clinical and Biological Sciences, University of Torino, 10100 Torino, Italy; ymera.pignochino@unito.it

2 Candiolo Cancer Institute, FPO-IRCCS, 10060 Candiolo, Italy; giovanni.crisafulli@unito.it (G.C.); giorgia.giordano@unito.it (G.G.); enrico.berrino@unito.it (E.B.); marialaura.centomo@unito.it (M.L.C.); silvia.brusco@unito.it (S.B.); marco.basirico@ircc.it (M.B.); elena.maldi@ircc.it (E.M.); alberto.pisacane@ircc.it (A.P.); valeria.leuci@ircc.it (V.L.); dario.sangiolo@unito.it (D.S.); LDambrosio@asl.at.it (L.D.); massimo.aglietta@ircc.it (M.A.); alberto.bardelli@unito.it (A.B.); giovanni.grignani@ircc.it (G.G.)

3 Department of Oncology, University of Torino, 10100 Torino, Italy

check for

updates

Citation: Pignochino, Y.; Crisafulli, G.; Giordano, G.; Merlini, A.; Berrino, E.; Centomo, M.L.; Chiabotto, G.; Brusco, S.; Basiricò, M.; Maldi, E.; et al. PARP1 Inhibitor and Trabectedin Combination Does Not Increase Tumor Mutational Burden in Advanced Sarcomas-A Preclinical and Translational Study. Cancers 2021, 13, 6295. https://doi.org/10.3390/ cancers13246295

Academic Editor: Armita Bahrami

Received: 22 November 2021 Accepted: 13 December 2021 Published: 15 December 2021

Publisher's Note: MDPI stays neutral with regard to jurisdictional claims in published maps and institutional affiliations.

Copyright: (c) 2021 by the authors. Licensee MDPI, Basel, Switzerland. This article is an open access article distributed under the terms and conditions of the Creative Commons Attribution (CC BY) license (https:// creativecommons.org/licenses/by/ $4.0 /)$.
4 Department of Medical Sciences, University of Torino, 10100 Torino, Italy; giulia.chiabotto@unito.it

5 Cardinal Massaia Hospital, 14100 Asti, Italy

6 Sarcoma Unit, Mannheim University Medical Center, University of Heidelberg, 68167 Mannheim, Germany; bernd.kasper@medma.uni-heidelberg.de

* Correspondence: alessandra.merlini@unito.it; Tel.: +39-0119933623

+ Co-first authors.

Simple Summary: Immunotherapy has revolutionized cancer treatment, but not for all tumor types. Indeed, sarcomas are considered "immune-cold" tumors, which are relatively unresponsive to immunotherapy. One strategy to potentiate immunotherapy efficacy is to increase tumor immunogenicity, for instance by boosting the number of candidate targets (neoantigens) to be recognized by the immune system. Tumor mutational burden indicates the number of somatic mutations identified in the tumor and normalized per megabase. Tumor mutational burden is considered as an acceptable, measurable surrogate of tumor neoantigens. Here, we explored whether the combination of two DNA-damaging agents, trabectedin and olaparib, could increase tumor mutational burden in sarcomas, to prime subsequent immunotherapy. We found no variation in tumor mutational burden after trabectedin + olaparib in preclinical and clinical samples. Therefore, other aspects should be considered to increase sarcoma immunogenicity, by exploiting different pathways such as the potential modulation of the tumor microenvironment induced by trabectedin + olaparib.

Abstract: Drug-induced tumor mutational burden (TMB) may contribute to unleashing the immune response in relatively "immune-cold" tumors, such as sarcomas. We previously showed that PARP1 inhibition perpetuates the DNA damage induced by the chemotherapeutic agent trabectedin in both preclinical models and sarcoma patients. In the present work, we explored acquired genetic changes in DNA repair genes, mutational signatures, and TMB in a translational platform composed of cell lines, xenografts, and tumor samples from patients treated with trabectedin and olaparib combination, compared to cells treated with temozolomide, an alkylating agent that induces hypermutation. Whole-exome and targeted panel sequencing data analyses revealed that three cycles of trabectedin and olaparib combination neither affected the mutational profiles, DNA repair gene status, or copy number alterations, nor increased TMB both in homologous recombinant-defective and proficient cells or in xenografts. Moreover, TMB was not increased in tumor specimens derived from trabectedinand olaparib-treated patients (5-6 cycles) when compared to pre-treatment biopsies. Conversely, repeated treatments with temozolomide induced a massive TMB increase in the SJSA- 1 osteosarcoma model. In conclusion, a trabectedin and olaparib combination did not show mutagenic effects and 
is unlikely to prime subsequent immune-therapeutic interventions based on TMB increase. On the other hand, these findings are reassuring in the increasing warning of treatment-induced hematologic malignancies correlated to PARP1 inhibitor use.

Keywords: advanced sarcomas; tumor mutational burden; DNA damage response and repair genes; trabectedin; olaparib; mutational signatures

\section{Introduction}

Immune-checkpoint inhibitors (ICI) have demonstrated a striking degree of efficacy in several advanced tumors, prompting a dedicated effort to identify predictive factors of response [1]. As of today, immune-infiltrate, T-cell receptor clonality, specific gene expression signatures have been associated with clinical response [2-4]. Moreover, tumor mutational burden (TMB), defined as the number of somatic mutations normalized per megabase, correlates with ICI activity [5,6], supposedly because it leads to the production of a larger set of tumor neoantigens, increasing the probability of triggering a specific T-cell clonal response. In this perspective, drugs generating DNA damage might induce mutations resulting in the production of neoantigens [7-9], despite the fact that these neoantigens may be related to subclones of the tumor (branched) and not to clonal mutations (truncal) that are more evenly expressed on all tumor cells and are correlated with a better response to ICI [10]. In the context of pharmacologically induced DNA damage, we focused on PARP1 inhibitors (PARP1i). Regardless of their use, PARP1i were developed to hit tumor cells bearing genetic (germinal or somatic) DNA damage response/repair defects or chemotherapy-induced ones [11]. Indeed, impairment of base excision, nucleotide excision and homologous repair mechanisms promotes the activation of the error-prone non-homologous end-joining (NHEJ) DNA repair machinery, eventually favoring the accumulation of mutations in tumor cells [12]. Inactivating mutations of key components in DNA damage response and repair (DDRR) pathways can lead to high TMB in tumor cells $[13,14]$. For instance, in ovarian cancers bearing BRCA1/2 defects, TMB is higher if compared to BRCA1/2 wild-type tumors [15]. Moreover, PARP1i stimulates immunomodulatory pathways in cancer cells and may reshape the tumor microenvironment [16]. These findings have raised interest in the potential of combining PARP1i with ICI; several trials are ongoing to explore this hypothesis [17]. The dark side of a TMB increase is the potential induction of secondary tumors, especially hematological ones. Indeed, the wider use of PARP1i brought about a small, but significantly increased, risk of developing myelodysplastic syndromes or leukemia, as evidenced by randomized clinical trials and a recent meta-analysis $[18,19]$.

In search of clinically feasible PARP1i partners, we and others showed that the antitumor activity of trabectedin was increased by PARP1 inhibition in sarcoma preclinical models [20-22]. Thereafter, we ran a phase $\mathrm{Ib}$ trial on the combination of trabectedin and olaparib in patients affected by advanced bone and soft tissue sarcomas, proving feasibility and showing preliminary signs of activity [23]. Since bone and soft tissue sarcomas are characterized by an averagely low TMB and fail to display significant sensitivity to ICI [24], we investigated whether this combination might increase TMB, so as to represent a priming intervention for further integration with immunotherapy, thanks to the appearance of tumor-specific neoantigens capable of activating T-cell responses. In this work, we evaluated DDRR mutational status, mutational signatures, and TMB after treatment with trabectedin and olaparib in a preclinical and translational platform of bone and soft tissue sarcoma cell lines, xenografts models, BRCA-proficient and defective cells and in temozolomide-treated cells as a positive control. Moreover, we took advantage of tumor samples derived from patients enrolled in the phase Ib TOMAS trial testing the trabectedin and olaparib combination in advanced sarcomas to further confirm our preclinical findings. We showed that the trabectedin and olaparib combination did not affect mutational signatures, DDRR gene status, gene copy number, clonality, and TMB. These findings indicate 
that trabectedin and olaparib combination did not show mutagenic effects and is unlikely to prime subsequent immune-therapeutic interventions based on TMB increase, while potentially reassuring the warning of myelodysplastic risk.

\section{Materials and Methods}

\subsection{Cell Cultures, BRCA1 Stable Silencing and Drug Treatment}

Human osteosarcoma (SJSA-1, CRL-2098, doubling time: 26 h), fibrosarcoma (HT-1080, CRL-121 doubling time: $22 \mathrm{~h}$ ) and ovarian cancer (A2780, UWB1.289_BRCA1, OVCAR-8 and IGROV-1 doubling time: 22, 30, 25, $26 \mathrm{~h}$, respectively) cell lines were obtained from the American Type Culture Collection (ATCC) and cultured following ATCC instructions. Human leiomyosarcoma cell line (DMR) was kindly provided by the group of Paola Spessotto (CRO-IRCCS, Aviano, Italy). One human liposarcoma cell line (402.91, doubling time: $21 \mathrm{~h}$ ) was kindly provided by Dr. Pierre Aman (University of Gothenburg, Gothenburg, Sweden) and cultured in RPMI medium supplemented with $10 \%$ FBS. We obtained 402.91 shBRCA1 (doubling time $26 \mathrm{~h}$ ) after infection with lentiviral particles, produced as described elsewhere [22]. Briefly, Dharmacon GIPZ ${ }^{\mathrm{TM}}$ Human BRCA1 shRNA Clone ID: V2LHS_280394 RHS4430-2001973 glycerol stock (Carlo Erba Reagents, Cornaredo, MI, Italy) was amplified in LB broth and selected by Ampicillin in LB-agar. The picked colony was amplified in LB broth and plasmids were purified by Qiagen Plasmids Maxy Prep Kit (Qiagen, Hilden, Germany). Packaging vectors pMDLg/pRRE pRSV-REV and pMD2.VSVG were used for lentiviral preparation. Efficiency of transduction was confirmed by detecting the percentage of GFP-positive cells using Cyan ADP Flow cytometer (Beckman Coulter, Brea, CA, USA) and by Western blot analysis (primary antibody: anti human BRCA1 \#9010 Cell Signaling Technologies, Leiden, The Netherlands). Trabectedin (PharmaMar, Madrid, Spain), temozolomide (T2577, Sigma Aldrich, Merck, Darmstadt, Germany) and olaparib (SelleckChem, Munich, Germany) were dissolved in DMSO and stored at $-80^{\circ} \mathrm{C}$ until use. Untreated cell lines (time 0 : T0) were subdued until cell viability assays for determining the concentration inhibiting $50 \%$ of cell viability (IC50) and to DNA extraction for whole exome sequencing (WES) analysis. Fifteen million cells were plated into 3 dishes of $150 \mathrm{~mm}$ diameter and after $24 \mathrm{~h}$ were treated with trabectedin, trabectedin plus olaparib or temozolomide at established IC50 for $72 \mathrm{~h}$ or left untreated. The treatment was repeated three times and if sufficient viable cells were spared by the drug treatment (at least $1 \%$ viable cells $=1.5 \times 10^{5}$ viable cells), DNA was extracted and prepared for WES (T1). The surviving fraction was subdued in another cell viability assay to verify the occurrence of drug resistance and to establish the novel IC50 (IC50'). Resistant cells were subdued to another 3 cycles of treatment with the novel IC50' and the surviving fraction underwent DNA extraction and WES analysis (T2) (Figure 1). 

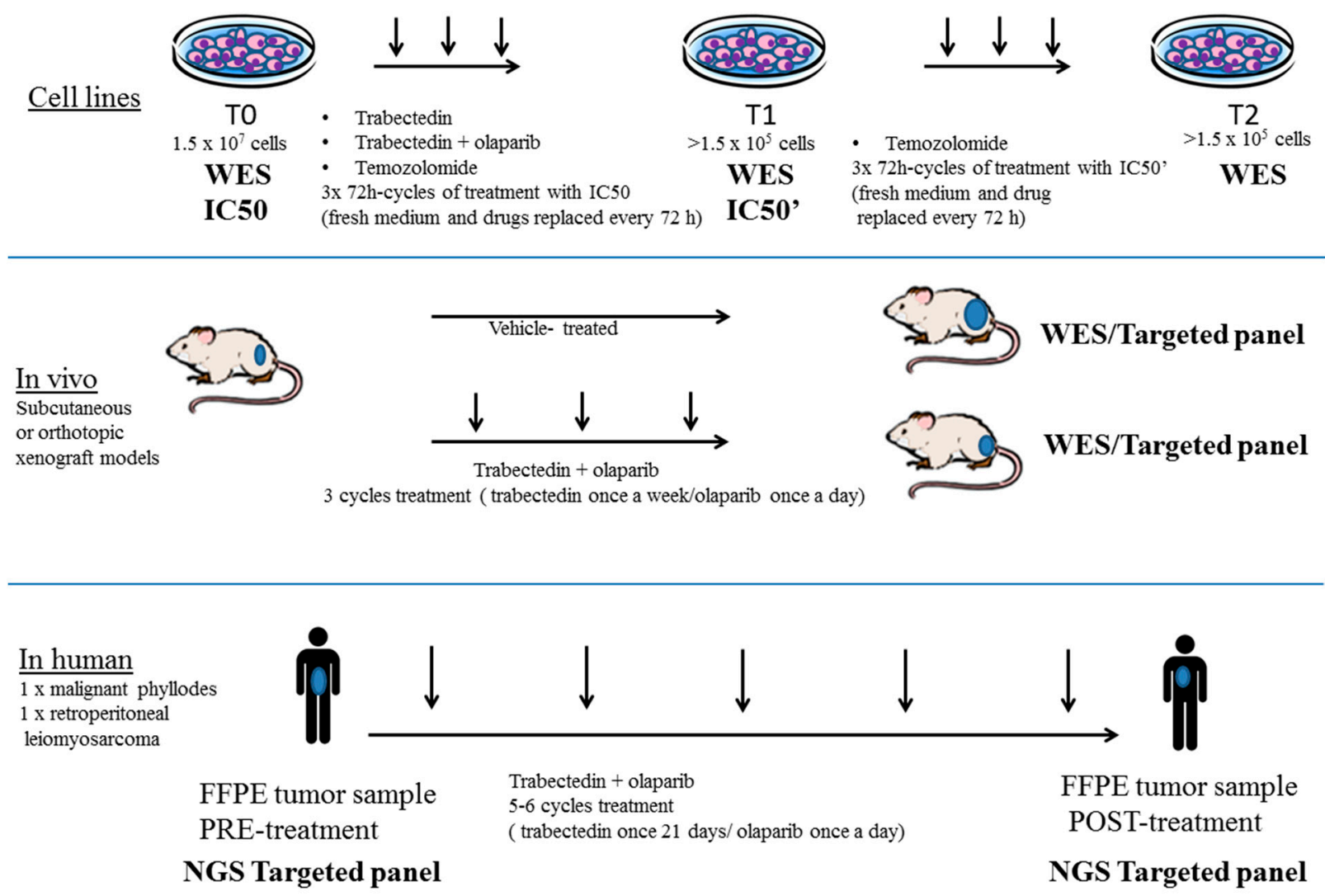

Figure 1. Experimental workflow on preclinical and translational platform. In vitro models $(n=9$ for trabectedin + olaparib treatment): cell lines underwent 3 cycles of drug treatment as described in the Methods section followed by DNA extraction and WES analyses (T1). After 3 cycles of treatment (T1) no sufficient cells survived trabectedin and olaparib treatment. Temozolomide-treated cells $(n=5)$ underwent another cell viability test, showing increased resistance. After establishing a higher IC50 $\left(\mathrm{IC} 50^{\prime}\right)$, surviving cells $(n=1)$ underwent 3 novel cycles of temozolomide treatment, followed by DNA extraction (T2). In vivo models $(n=3)$ : DMR, HT1080 and SJSA-1 were injected into the right flank or into the uterine wall (orthotopic model: DMR only) of NOD/SCID mice. After tumor engraftment, mice were treated with trabectedin + olaparib for 3 weeks or left untreated. Patient samples $(n=2)$ : two patients enrolled in the TOMAS trial, affected by advanced sarcomas, were treated with trabectedin and olaparib combination at the recommended phase 2 dose. Extracted DNA (pre and post treatment) was analyzed by next-generation sequencing approaches: WES or targeted panel (Oncomine TML) scalable in low DNA concentration and FFPE conditions.

\subsection{Cell Viability Assay and IC50 Determination}

To determine the concentration inhibiting $50 \%$ of cell viability (IC50) of temozolomide or a trabectedin and olaparib combination, viability assays were performed using a Cell Titer-Glo ${ }^{\circledR}$ kit (Promega Corporation Madison, WI, USA) after $72 \mathrm{~h}$ of drug treatment. Briefly, 2000 cells were seeded on 96-well plates and incubated for $24 \mathrm{~h}$ in their complete medium and subsequently for $72 \mathrm{~h}$ with scalar doses of temozolomide $(1-0.0625 \mathrm{mM})$ or trabectedin $(1-0.0625 \mathrm{nM})$ and olaparib $(10-0.625 \mu \mathrm{M})$ as single agents or as constant combination. Cell Titer-Glo ${ }^{\circledR}$ Reagent was added to each well for $15 \mathrm{~min}$ at room temperature in the dark to allow luminescence signal production. Synergy HT luminometer (Biotek Instruments, Winooski, VT, USA) and Gen5 v1.09 software (Biotek Instruments) were used for signal acquisition. IC50 with its 95\% confidence intervals (95\% CI), and drug synergism, expressed as combination index (CI) calculated at IC50 with its estimated standard deviation were obtained by using CalcuSyn software (Biosoft, Cambridge, UK) based on isobologram analysis. A dose-effect curve was obtained by GraphPad Prism 7.0.2 software (La Jolla, CA, USA) and analyzed by two-way ANOVA with Bonferroni's correction. 


\subsection{Western Blot}

To obtain total protein extracts, cells were detached with trypsin-EDTA in PBS (EuroClone, Pero, Milan, Italy), washed with cold PBS, and re-suspended at a concentration of $10^{7}$ cells/mL in Lysis Buffer 6 (R\&D Systems, Minneapolis, MN, USA). Cells were mechanically lysed on ice with a pipette and rocked gently for $20 \mathrm{~min}$ at $4{ }^{\circ} \mathrm{C}$ and then clarified to remove cell debris. Protein concentration was determined by using a bicinchoninic acid assay (BCA Protein Assay, Thermo Fisher Scientific, Life Technology Italia, Monza, Italy) and absorbance levels were measured using the BioPhotometer (Eppendorf, Hamburg, Germany). A total of $30 \mu \mathrm{g}$ of protein samples were resolved by electrophoresis on $4-20 \%$ mini-PROTEAN ${ }^{\circledR}$ TGX ${ }^{\mathrm{TM}}$ PreCast gels (Bio-Rad Laboratories, Hercules, CA, USA), and then transferred to a nitrocellulose membrane via the Trans-Blot ${ }^{\circledR}$ Turbo $^{\mathrm{TM}}$ Transfer System (Bio-Rad Laboratories). Non-specific sites were blocked with $5 \%$ milk for $1 \mathrm{~h}$ at room temperature after which the membranes were incubated overnight at $4{ }^{\circ} \mathrm{C}$ with anti-human BRCA1 (1/1000 dilution in TBS/Tween 0.2\%/BSA 0.5\%; Cell Signaling Technology, Leiden, The Netherlands) or anti-human $\beta$-Actin (1/1000 dilution TBS/Tween $0.2 \%$ /BSA $0.5 \%$; Cell Signaling Technology, Leiden, The Netherlands) antibodies. Specific signals were visualized with HRP-conjugated secondary antibodies (Jackson Immuno Research Laboratories, West Grove, PA, USA) and detected using the Bio-Rad Chemidoc ${ }^{\mathrm{TM}}$ Touch Imaging System (Bio-Rad Laboratories) following exposure to Clarity ${ }^{\mathrm{TM}}$ Western ECL substrate (Bio-Rad Laboratories). Band intensity was quantified by ImageLab v5.2.1 (Bio-Rad Laboratories) using $\beta$-actin as normalizers.

\subsection{Sarcoma Xenograft Models in NOD/SCID Mice}

One million 402.91, 402.91shBRCA1, DMR, HT1080 and SJSA-1 cells were injected subcutaneously into the right flank or orthotopically in the uterine wall $\left(4 \times 10^{5} \mathrm{DMR}\right)$ of 5 -week-old female NOD/SCID mice. After tumor engraftment, mice were treated with three cycles of $25 \mathrm{mg} / \mathrm{kg} /$ day olaparib (by intraperitoneal injection, 5 days/week) and $0.05 \mathrm{mg} / \mathrm{kg}$ trabectedin (intravenous administration once a week) or left untreated for 21 days (17 for SJSA) as previously described [22] accordingly to protocols approved by the Institutional Ethics Review Board (IRB) and by the Italian Ministry of Health (Aut. Min. 178/2015-PR). At the end of the experiments, tumor tissues were snap-frozen in liquid nitrogen for further DNA extraction.

\subsection{TOMAS Trial Patients}

Two patients affected by advanced sarcomas (one relapsing retroperitoneal grade 3 leiomyosarcoma and one malignant phyllodes tumor with multiple lung metastases), progressing after anthracycline-based treatment and enrolled in the phase $1 \mathrm{~b}$ clinical trial TOMAS [23], were treated with trabectedin $1.1 \mathrm{mg} / \mathrm{m}^{2}$ every 3 weeks plus olaparib $150 \mathrm{mg}$ twice daily. After 5 and 6 cycles, respectively, both patients underwent surgery, having achieved a partial response according to RECIST 1.1. Archival FFPE samples at baseline and after surgery were collected for molecular analyses according to the TOMAS protocol.

\subsection{DNA Extraction}

Total DNA was extracted from cultured cells (at least $1.5 \times 10^{5}$ cells) and from frozen tissues using the Maxwell ${ }^{\circledR}$ RSC Blood DNA Kit AS1400 (Promega), from FFPE archival tumor biopsy and surgical excision, after microdissection of the tumor area, using the Maxwell ${ }^{\circledR}$ RSC DNA FFPE Kit AS1450 (Promega), and the RSC Maxwell Instrument (Promega) in accordance with the manufacturer's instructions. DNA purity was checked by NanoDropTM (Thermo Fisher Scientific, Life Technology Italia, Monza, Italy). DNA concentration was determined by the Qubit dsDNA HS (high sensitivity) assay kit (Thermo Fisher Scientific, Life Technology Italia) and the Qubit ${ }^{\circledR}$ 3.0 Fluorometer (Thermo Fisher Scientific, Life Technology Italia). DNA fragmentation was assessed by gel electrophoresis and a 2100 Bioanalyzer High Sensitivity DNA assay Kit (Agilent Technologies, Agilent Technologies, Inc., Santa Clara, CA, USA). 


\subsection{Genetic Analyses after Whole Exome Sequencing and NGS Targeted Approach}

Library preparation for whole exome sequencing analysis was performed using a minimum of $300 \mathrm{ng}$ of DNA from tissue samples and cell lines. The library preparation was performed by IntegraGen SA (Evry, France) using the Twist Bioscience ${ }^{\circledR}$ (South San Francisco, California, USA) Human Core Exome (Consensus CDS) + IntegraGen content, for a genomic target of $37 \mathrm{Mb}$. All libraries were sequenced on a Novaseq 6000 sequencer (Illumina) and 100-150 bp paired-end reads were generated with an average sequencing depth of 135X depth per exome and a coverage $>98 \%$ at $>50 X$. Genetic discovery analysis was performed by an in-house NGS pipeline $[25,26]$ constructed for WES analyses of paired cancer genomes in order to call somatic variations, indels and copy number alterations. The Xenome tool [27] was used to evaluate mouse-derived reads and to remove mouse sequences in xenograft samples. Human-assigned reads were then mapped to assemble hg19 of the human reference genome using a BWA-mem algorithm with standard parameters. PCR duplicates were removed using the RMDUP command of SAM tools package. Reads with more than 3 mismatches and bases with a Phred Score $<30$ were filtered out. All mutations detected by reads as having a strand bias or exclusively by mismatch in head/tail of the read were discarded. Only mutations with a minimum depth of $5 \mathrm{X}$ and at least $10 \%$ allelic frequency were considered. Reads supporting MUC6 and MUC2 gene variations were filtered out because DNA sequences of the human mucin genes have not been completely resolved due to the repetitive nature of their central exon coding for Proline, Threonine and Serine-rich sequences [28]. BAM files were then analyzed by comparing pre-treatment and post-treatment samples to identify mutations and indels in post-treatment matched samples. Mutations and indels common to both samples were classified as pre-treatment, while alterations present in post-treatment but not in pre-treatment were classified as acquired. After verifying the quality control of sequencing parameters for all samples, we confirmed that the samples analyzed belonged to the matched post-treatment samples by comparing their single nucleotide polymorphisms. The identity of all cell lines and xenografts were confirmed using the unique allelic profile (based on Single Nucleotide Polymorphism Identification, SNP_ID) using the dbSNP-version 147. Only alleles with a fractional abundance above $20 \%$ and depth above $10 \mathrm{X}$ were considered. For annotation of germinal alterations of 341 DDRR genes, we filtered out synonymous mutations and mutations with fractional abundance less than $10 \%$. PolyPhen- 2 was used to annotate the prediction effect of the mutation on the protein coded by the genes. TMB was calculated from WES data, taking into consideration somatic mutations after data normalization on the specific covered target region of each sample [29,30]. Our methodology was set up on biopsies of metastatic colon tissue, as previously described [26,29-31]. The ratio of median gene depth to the median depth of the whole exome was defined as Gene Copy Number (GCN). For each gene, its GCN in the pre- and post-treatment samples and the corresponding copy number variation (ratio between matched GCNs) were reported. A circular binary segmentation (CBS) algorithm using DNAcopy R module was performed to cluster the gene copy-number alterations [25]. Samples purified from HT1080 cells, DMR uterine orthotopic and HT1080 subcutaneous xenografts, and FFPE tumor samples from TOMAS trial patients underwent targeted panel sequencing with Oncomine ${ }^{\mathrm{TM}}$ Tumor Mutation Load Assay (Thermo Fisher Scientific, Inc., Waltham, MA, USA). This ampliconbased target gene panel (409 genes) was properly designed to evaluate the TMB even in fragmented and usually impracticable FFPE DNA samples. The NGS libraries were built starting from a total of $20 \mathrm{ng}$ of DNA using the IonChef ${ }^{\mathrm{TM}}$ System (Thermo Fisher Scientific, Life Technology Italia, Monza, Italy). Briefly, eight samples per run were loaded in a preparation plate containing the IonCode ${ }^{\mathrm{TM}}$ barcodes. The automated instrument performed 16 cycles of PCR with 16 min of annealing and extension. Eight prepared libraries were diluted to $50 \mathrm{pM}$, pooled and loaded on the Ion $540^{\mathrm{TM}}$ chip by means of the IonChef ${ }^{\mathrm{TM}}$ System. Sequencing was achieved using the Ion GeneStudio ${ }^{\mathrm{TM}}$ S5 Plus System (Thermo Fisher Scientific, Life Technology Italia, Monza, Italy). The panel results were optimized for a total of 8 to 10 million reads with a 500X median coverage. The reads 
were aligned to assemble hg19 of the human reference genome by the Torrent Suite ${ }^{\mathrm{TM}} \mathrm{v} 5.8$ (Thermo Fisher Scientific, Life Technology Italia, Monza, Italy), generating the BAM files, which were transferred on the Ion Reporter Server ${ }^{\mathrm{TM}} \mathrm{v} 5.10$ (Thermo Fisher Scientific, Life Technology Italia, Monza, Italy) for the TMB calculation using the Oncomine ${ }^{\mathrm{TM}}$ Tumor Mutation Load v2.0 workflow (Thermo Fisher Scientific, Life Technology Italia, Monza, Italy). To standardize the analyses and to reduce the impact of sequencing artifacts derived from the formalin fixation, we set the allele frequency limit of detection at $10 \%$ for all the samples. The TMB was calculated as the number of single nucleotide variants, small indel non-synonymous mutations divided with at least 60X of alternative read depth by the active genome (regions under 250X were excluded). Germline variants were filtered automatically by cross-referencing with UCSC common SNPs, ExAC, 10,000 Genomes, and 5000Exomes databases.

\subsection{Mutational Signature Analyses}

The analysis of mutational signatures using the "fitting" method can identify the best combination of the known signatures explaining the observed mutational profile of cell lines and xenografts. Many of these signatures have been associated with a defined etiology, including mutational profiles derived from exposure to different treatments. Genetic alterations with fractional abundance higher than $10 \%$ were used for mutational signature analysis. The profile of each genetic signature was calculated using the six substitution subtypes: $\mathrm{C}>\mathrm{A}, \mathrm{C}>\mathrm{G}, \mathrm{C}>\mathrm{T}, \mathrm{T}>\mathrm{A}, \mathrm{T}>\mathrm{C}, \mathrm{T}>\mathrm{G}$. In detail, all substitutions were referred to the pyrimidine of the mutated Watson-Crick base pair. The flanking $5^{\prime}$ and $3^{\prime}$ bases of each mutation were characterized and reported in a mutational catalogue using sigprofilerMatrixGenerator [32], as initially reported in [33,34]. Using the information of single nucleotide variants in the matrix, a series of mutational profiles were extracted, and genetic signatures were calculated using the MuSiCa tool [35] and Mutational Patterns package [36]. As reported, the mutational catalogue of each sample (M) may be considered as the composite of all the mutagenic processes that were active in the cancer cells. Then, mutational signature fitting estimated the contribution (E) of each mutational process specifying the percentage of mutations that could be attributed to each genetic signature (S) for each cell line/xenograft (such as $\mathrm{M} \approx \mathrm{S} \times \mathrm{E}$ ). The reference $\mathrm{v} 2.0$ of the COSMIC signature database was used for fitting analysis. Cosine similarity was calculated by MuSiCa [35] and Mutational Patterns tools [36] and used to evaluate the similarity between the original mutational catalogue of profiles (identified by the mutations in the sample) and the reconstructed mutational profile based on the optimal linear combination of all COSMIC signatures (identified after fitting) [36].

\section{Results}

3.1. Generation of a Preclinical and Translational Platform of Bone and Soft Tissue Sarcoma Cell Lines, Xenografts and Tumor Samples from Patients Treated with Trabectedin and Olaparib

We generated a panel of bone and soft tissue sarcoma cell lines comprising one leiomyosarcoma (DMR), one liposarcoma (402.91), one osteosarcoma (SJSA-1) and one fibrosarcoma (HT-1080) treated with at least three cycles of trabectedin and olaparib combination or with temozolomide as a control (as described in the Methods section), obtaining a cell surviving fraction $(>1 \%)$ sufficient for DNA extraction and WES analysis $\left(>1.5 \times 10^{5}\right.$ viable cells, Figure 1).

In order to include a BRCA1 defective control, we generated a stable, silenced BRCA1 402.91 liposarcoma cell line (402.91 shBRCA1) and we completed the panel with BRCA1 defective (IGROV-1 and OVCAR-8) and BRCA1 proficient (A2780 and UWB 1.289_BRCA1) ovarian cancer cell lines. The expression of BRCA1 protein in the aforementioned cell lines was shown in Figure 2 and in Figure S1. 
$\mathbf{A}$

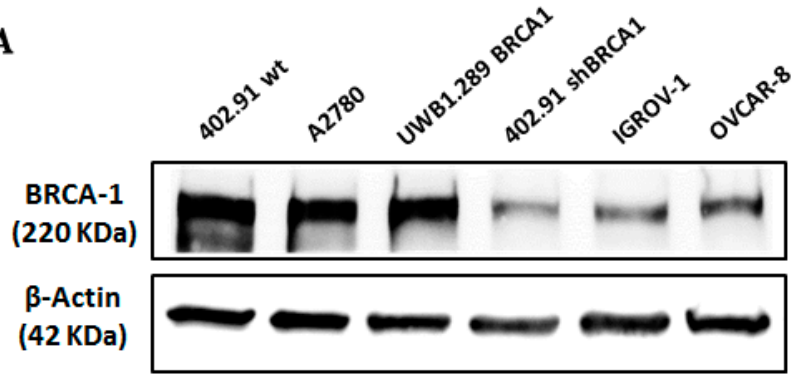

B

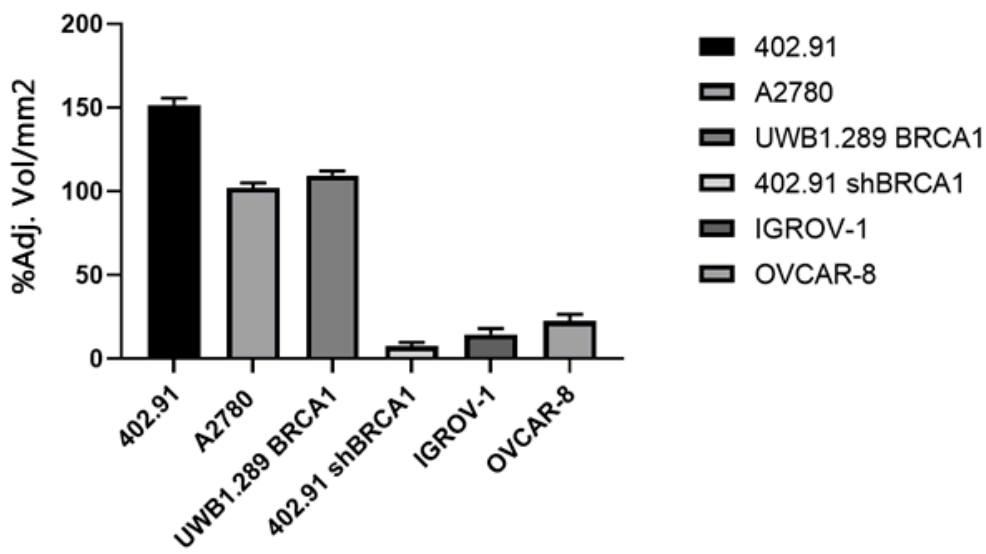

Figure 2. BRCA1 protein expression in cell lines. (A) Representative Western blot analysis of BRCA1 expression in BRCA1 proficient (402.91 wild type (wt), A2780, UWB1.289 BRCA1) and BRCA1 defective cells (BRCA1 stable silenced 402.91 (402.91 shBRCA1), IGROV-1 and OVCAR-8). $\beta$-actin was used as loading controls. (B) Densitometric analysis of protein band intensity normalized on housekeeping controls expressed as mean value of three replicates \pm standard deviations of the percentage of adjusted volume $/ \mathrm{mm}^{2}$.

In Table 1 , we summarized the drug concentration inhibiting $50 \%$ of cell viability for each cell line established after $72 \mathrm{~h}$ of treatment (72 h IC50). In particular, we observed that 402.91 and DMR cells were the most sensitive to trabectedin and olaparib combination followed by SJSA-1, while HT-1080 cells were the most resistant ones.

Table 1. Concentration inhibiting 50\% of cell viability (IC50) and 95\% confidence intervals (95\%CI).

\begin{tabular}{|c|c|c|c|c|c|}
\hline \multirow[b]{2}{*}{ Cell Line } & \multicolumn{2}{|c|}{ IC50 Trabectedin $(95 \% \mathrm{CI}) \mathrm{nM}$} & \multicolumn{2}{|c|}{ IC50 Olaparib (95\% CI) $\mu \mathrm{M}$} & \multirow{2}{*}{$\begin{array}{c}\text { Combination } \\
\text { Combination } \\
\text { Index } \pm \text { Est. SD }\end{array}$} \\
\hline & as SINGLE Agent & $\begin{array}{l}\text { in Combination } \\
\text { with Olaparib }\end{array}$ & as Single Agent & $\begin{array}{l}\text { in Combination } \\
\text { with Trabectedin }\end{array}$ & \\
\hline DMR & $0.57(0.45-0.64)$ & $0.13(0.12-0.14)$ & $9.02(8.11-10.23)$ & $1.30(1.18-1.43)$ & $0.47 \pm 0.06$ \\
\hline SJSA-1 & $0.25(0.16-0.39)$ & $0.18(0.15-0.19)$ & $14.55(6.66-29.64)$ & $1.80(1.52-1.93)$ & $0.86 \pm 0.11$ \\
\hline HТ-1080 & $0.75(0.68-0.81)$ & $0.60(0.59-0.64)$ & $>20$ & $6.04(5.92-6.42)$ & $0.89 \pm 0.15$ \\
\hline 402.91 & $0.46(0.37-0.54)$ & $0.12(0.11-0.14)$ & $7.12(5.53-7.91)$ & $1.84(1.37-2.68)$ & $0.50 \pm 0.12$ \\
\hline 402.91 shBRCA1 & $0.19(0.12-0.28)$ & $0.14(0.04-0.53)$ & $2.92(2.19-3.89)$ & $1.40(0.38-5.27)$ & $1.24 \pm 0.22$ \\
\hline A2780 & $0.41(0.29-0.58)$ & $0.20(0.12-0.32)$ & $8.73(7.22-10.55)$ & $1.97(1.22-3.18)$ & $0.7 \pm 0.11$ \\
\hline UWB1.289 BRCA1 & $0.44(0.30-0.66)$ & $0.41(0.37-0.47)$ & $10.36(5.37-19.98)$ & $4.18(3.67-4.75)$ & $1.35 \pm 0.17$ \\
\hline OVCAR-8 & $0.38(0.12-1.20)$ & $0.16(0.04-0.70)$ & $9.77(6.06-15.74)$ & $1.62(0.37-7.04)$ & $0.60 \pm 0.18$ \\
\hline IGROV-1 & $0.62(0.39-0.99)$ & $0.326(0.18-0.58)$ & $21.04(13.59-32.57)$ & $3.26(1.82-5.85)$ & $0.68 \pm 0.14$ \\
\hline
\end{tabular}

Combination index \pm estimated standard deviations (Est. SD) calculated at IC50 for trabectedin and olaparib combination in cell lines of different histotypes: leiomyosarcoma (DMR), osteosarcoma (SJSA-1), fibrosarcoma (HT-1080), liposarcoma (wild-type 402.91 and shBRCA1 402.91) and ovarian cancer (A2780, UWB1.289 BRCA1, OVCAR-8, IGROV-1). IC50 was calculated for each cell line after $72 \mathrm{~h}$ of treatment with serial dilutions of trabectedin $(1-0.125 \mathrm{nM})$ and olaparib $(10-1.25 \mu \mathrm{M})$ as single agents or in combination (constant ratio 1:10,000). Each experiment consisted of eight technical replicates and was repeated three times. 
We observed that the sensitivity to trabectedin and olaparib as single agents was higher for 402.91shBRCA1 cells when compared to their parental counterpart (402.91; Table 1). Of note, in 402.91shBRCA1 cells, these two drugs did not have a synergistic effect (combination index $>1$ ), possibly due to the high activity of both single agents. As shown in Table 1, IC50 values of trabectedin and olaparib combination varied among different ovarian cell lines. Each cell line was treated with the established $72 \mathrm{~h}$ IC50 of the combination. Surviving fractions were collected at the end of the third cycle of treatment and underwent DNA extraction and WES (T1). No cell lines but SJSA-1 gave rise to sufficient surviving fraction $\left(>1.5 \times 10^{5}\right.$ viable cells), to be challenged with further cycles of treatment with trabectedin and olaparib. We challenged all sarcoma cell lines with temozolomide and, once again, SJSA-1 was the least sensitive to temozolomide (Table 2). In particular, SJSA-1 cells' treatment with the established temozolomide $72 \mathrm{~h}$ IC50 $(677 \mu \mathrm{M})$ gave sufficient surviving fraction $\left(>1.5 \times 10^{5}\right.$ viable cells $)$ after the third cycle of treatment (T1). This fraction (now referred as SJSA-1 T1 cells) underwent further cell viability assays, showing increased drug resistance. Namely, the $72 \mathrm{~h} \mathrm{IC50}$ was $677 \mu \mathrm{M}$ with a $95 \%$ confidence interval ranging from 486 to $943 \mu \mathrm{M}$ in wild-type SJSA-1 and $1499 \mu \mathrm{M}$ (IC50') with a 95\% confidence interval ranging from 1051 to $2137 \mu \mathrm{M}$ in SJSA-1 T1 cells (Table 2).

Table 2. IC50 and 95\% CI after 72 h-temozolomide treatment.

\begin{tabular}{cc}
\hline Cell Line & IC50 Temozolomide (95\% CI) $\boldsymbol{\mu M}$ \\
\hline DMR & $594(499-706)$ \\
HT-1080 & $489(416-575)$ \\
402.91 & $151(133-172)$ \\
402.91 shBRCA1 & $242(208-281)$ \\
SJSA-1 & $677(486-943)$ \\
SJSA-1 T1 & $1499(1051-2137)$ \\
\hline
\end{tabular}

Concentration inhibiting $50 \%$ of cell viability (IC50) after $72 \mathrm{~h}$ of treatment with serial dilutions of temozolomide $(2000-125 \mu \mathrm{M})$ as single agents in sarcoma cell lines of different histotypes: leiomyosarcoma (DMR), osteosarcoma (SJSA-1), fibrosarcoma (HT-1080), liposarcoma (wild-type 402.91 and shBRCA1 402.91. SJSA-1 T1 cells were generated from SJSA-1 after three cycles of $72 \mathrm{~h}$ treatment with $677 \mu \mathrm{M}$ of temozolomide. Each experiment consisted of eight technical replicates and was repeated three times.

The SJSA-1 T1 cells were further treated with $3 \times 72$ h cycles of treatment, with temozolomide at IC50' $(1499 \mu \mathrm{M})$ reaching the second time point of DNA extraction (T2).

The translational platform was further enriched with in vivo mouse models, derived from the human sarcoma cell lines tested in vitro. In particular, 402.91, 402.91shBRCA1, DMR, SJSA-1 and HT1080 cell lines were injected subcutaneously into the right flank of NOD/SCID mice. DMR cells were also implanted orthotopically into the uterine wall of an additional cohort of female NOD/SCID mice. All cell lines apart from 402.91 and 402.91shBRCA1 were successfully engrafted in NOD/SCID mice, and animals were treated with three cycles of trabectedin and olaparib or left untreated.

In the three successfully engrafted subcutaneous models (DMR, SJSA-1 and HT1080), the trabectedin and olaparib combination reduced tumor volume by 13.3, 2.8, and 1.4fold compared to untreated controls, respectively (Figure 3). In the DMR orthotopic model, the tumor growth was revealed by in vivo imaging techniques and the reduction induced by the combination was 2550 -fold of the photon intensity compared to untreated controls (Figure 3). No sign of toxicity was observed in terms of weight reduction and after gross necropsy.

To complete our preclinical and translational platform with tumor samples derived from patients treated with trabectedin and olaparib combination, we obtained FFPE tumor tissue before and after treatment. One metastatic malignant phyllode and one retroperitoneal leiomyosarcoma were treated with five and six cycles of trabectedin and olaparib combination, achieving partial responses and making them eligible to complete surgery. All 
samples before and after treatment were analyzed by NGS to investigate both mutational status of DDRR genes and TMB (Figure 1).

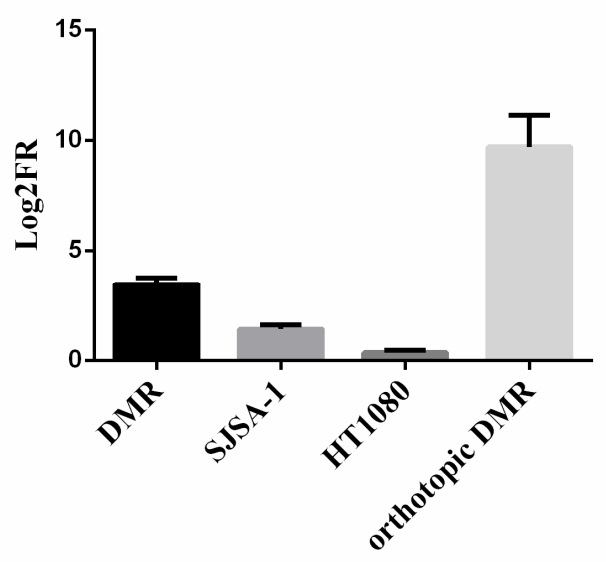

Figure 3. Antitumor effect in DMR, SJSA-1 and HT1080 xenograft models and DMR orthotopic model treated with trabectedin and olaparib combination compared to untreated controls. Five mice per group (control and combination treated) were included in the experiment. Log2FR $=\log 2$ fold-reduction in tumor volume as compared to untreated controls. Results are depicted as mean of $\log 2 \mathrm{FR} \pm$ standard deviation.

3.2. Genetic Analysis of DNA Damage Response and Repair (DDRR) Genes and Copy Number Alteration in a Preclinical and Translational Platform of Bone and Soft Tissue Sarcomas Treated with Trabectedin and Olaparib

We explored DDRR pathways by analyzing mutations and CNV after WES or targeted panel NGS in our preclinical and translational platform of bone and soft tissue sarcomas before and after the treatment with trabectedin and olaparib combination. After quality controls of all WES outputs, single nucleotide polymorphisms (as annotated in dbSNPs v.147) were analyzed comparing treated and untreated samples, confirming concordance (percentage higher than 95\% of concordance, Supplementary Table S1).

Based on Gene Ontology, we selected 341 DDRR genes (listed in Supplementary Table S2) for their role in the base excision repair (BER), DNA damage response (DDRR), Fanconi anemia homologous recombination (HRFA), nucleotide excision repair (NER), non-homologous end joining (NHEJ) and DNA mismatch repair (MMR) pathways and we investigated their mutational status. Moreover, we predicted the possible functional impact of identified amino acid substitutions on stability and function using the Polyphen-2 tool excluding common SNPs. In SJSA-1 cells, we observed three "probably damaging" nonsynonymous amino acid substitutions in BER genes (MUTYH, NEIL1 and NEIL2), five in DDRR genes (SENP7, INO80D, UBA7; UBR5, Y1AP1), eight in HRFA genes (CNTROB, DDX11, FANCD2, MCMDC2, NSMCE2), one in the MMR gene (RFC1), two in NER genes (XPC, BIVM-ERCC5). In DMR cells, two genes belonging to DDR (UPF1, TRRAP) displayed mutations and one gene (DCAF4L2), belonging to the NER pathway. In wild-type and shBRCA1 402.91 cells, we observed four mutations in DDR genes (CINP, HERC2, HUS1B, SENP7), five mutations in four HRFA genes (DDX11, NBN, PARP4, SLX4), one in the MMR gene (MSH4) and two in NER genes (DCAF4, MC1R). In one patient tumor sample (TOMAS B), mutations were found in both DDRR (TP53) and HRFA (WRN) pathways. No relevant modulation in the allelic frequency of the identified mutations were induced by trabectedin and olaparib treatment both in cell lines and in xenograft models, while a negligible increase in WRN mutations was observed in the sample derived from patient TOMAS B (Table 3). 


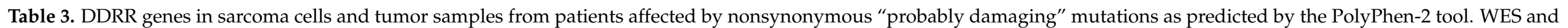
targeted panel sequencing results were analyzed searching for single nucleotide non-synonymous substitutions not previously described as SNP.

\begin{tabular}{|c|c|c|c|c|c|c|c|}
\hline \multirow[b]{2}{*}{ Pathway } & \multirow[b]{2}{*}{ Gene } & \multirow[b]{2}{*}{ Base Change } & \multirow{2}{*}{$\begin{array}{c}\text { Amino Acid } \\
\text { Change }\end{array}$} & \multicolumn{4}{|c|}{ Mean Allelic Frequency } \\
\hline & & & & SJSA-1 Untreated Cells & SJSA-1 TR + OL Treated Cells & SJSA-1 Untreated Xenografts & $\begin{array}{l}\text { SJSA-1 TR + OL } \\
\text { Treated Xenografts }\end{array}$ \\
\hline \multirow{3}{*}{ BER } & MUTYH & c.T538C & p.Y180H & 48.76 & 50.00 & 39.67 & 39.58 \\
\hline & NEIL1 & c.C826A & p.P276T & 48.28 & 36.73 & 52.94 & 40.60 \\
\hline & NEIL2 & c.C307T & p.R103W & 65.67 & 63.89 & 66.47 & 67.76 \\
\hline \multirow{4}{*}{ DDR } & SENP7 & c.G1909T & p.D637Y & 48.60 & 49.21 & 48.34 & 46.57 \\
\hline & INO80D & c.C2876G & p.S959C & 49.05 & 44.97 & 45.58 & 44.19 \\
\hline & UBR5 & c.G3850A & p.A1284T & 46.02 & 43.45 & 49.16 & 53.05 \\
\hline & $Y Y 1 A P 1$ & c.C385T & p.L129F & 40.85 & 42.10 & 48.72 & 41.65 \\
\hline \multirow{8}{*}{ HRFA } & CNTROB & c.G605T & p.R202L & 45.34 & 55.05 & 44.74 & 44.67 \\
\hline & CNTROB & c.G1316A & p.R439Q & 43.91 & 51.23 & 48.23 & 44.21 \\
\hline & DDX11 & c.C1040A & p.A347D & 37.70 & 42.21 & 38.87 & 34.82 \\
\hline & FANCD2 & c.T1367G & p.L456R & 26.66 & 25.67 & 25.12 & 24.77 \\
\hline & FANCD2 & c.A1868C & p.Q623P & 32.46 & 29.70 & 36.02 & 31.80 \\
\hline & $M C M D C 2$ & c.C1570T & p.P524S & 28.06 & 29.59 & 29.16 & 31.35 \\
\hline & NSMCE2 & c.T377G & p.F126C & 45.10 & 47.71 & 52.83 & 45.60 \\
\hline & SLX4IP & c.G779A & p.G260E & 46.67 & 38.86 & 41.86 & 48.35 \\
\hline MMR & RFC1 & c.G2076C & p.E692D & 100.00 & 98.00 & 100.00 & 98.72 \\
\hline \multirow{2}{*}{ NER } & $X P C$ & c.T860G & p.F287C & 53.38 & 46.67 & 50.49 & 49.13 \\
\hline & BIVM-ERCC5 & c.G2003A & p.R668H & 17.62 & 15.28 & 13.29 & 13.71 \\
\hline \multirow{2}{*}{ Pathway } & \multirow{2}{*}{ Gene } & \multirow{2}{*}{ Base Change } & \multirow{2}{*}{$\begin{array}{l}\text { Amino Acid } \\
\text { Change }\end{array}$} & \multicolumn{2}{|c|}{ Mean Allelic Frequency } & & \\
\hline & & & & DMR Untreated Cells & DMR TR + OL Treated Cells & & \\
\hline \multirow{2}{*}{ DDR } & UPF1 & c.G2204A & p.G735D & 100.00 & 98.56 & & \\
\hline & TRRAP & c.C2165T & p.S722F & 17.62 & 15.85 & & \\
\hline NER & $D C A F 4 L 2$ & c.G250T & p.D84Y & 53.38 & 47.24 & & \\
\hline
\end{tabular}


Table 3. Cont.

\begin{tabular}{|c|c|c|c|c|c|c|c|c|}
\hline \multirow[b]{2}{*}{ Pathway } & \multirow[b]{2}{*}{ Gene } & \multirow[b]{2}{*}{ Base Change } & \multirow{2}{*}{$\begin{array}{l}\text { Amino Acid } \\
\text { Change }\end{array}$} & \multicolumn{2}{|c|}{ Mean Allelic Frequency } & & & \\
\hline & & & & $\begin{array}{l}\text { TOMAS Untreated } \\
\text { Patient B }\end{array}$ & $\begin{array}{l}\text { TOMAS TR + OL } \\
\text { Treated Patient B }\end{array}$ & & & \\
\hline DDR & TP53 & c.G248A & p.S215N & 42.44 & 47.76 & & & \\
\hline HRFA & $W R N$ & c.G250T & p.D84Y & $<10 \%$ & 15.38 & & & \\
\hline \multirow{2}{*}{\multicolumn{2}{|c|}{ Pathway }} & \multirow[b]{2}{*}{ Gene } & \multirow[b]{2}{*}{ Base Change } & \multirow[b]{2}{*}{ Amino Acid Change } & \multicolumn{4}{|c|}{ Mean Allelic Frequency } \\
\hline & & & & & $\begin{array}{c}402.91 \\
\text { Untreated Cells }\end{array}$ & $\begin{array}{c}402.91 \\
\text { TR + OL Treated Cells }\end{array}$ & $\begin{array}{l}402.91 \text { shBRCA1 } \\
\text { Untreated Cells }\end{array}$ & $\begin{array}{c}\text { 402.91 shBRCA1 } \\
\text { TR + OL Treated Cells }\end{array}$ \\
\hline \multirow{4}{*}{\multicolumn{2}{|c|}{ DDR }} & CINP & c.C159G & p.N53K & 49.26 & 52.54 & 46.71 & 51.45 \\
\hline & & HERC2 & c.A32G & p.Q11R & 0 & 27.78 & 11.76 & 16.67 \\
\hline & & HUS1B & c.C390A & p.H130Q & 51.36 & 45.11 & 55.67 & 54.41 \\
\hline & & SENP7 & c.A1836T & p.Q612H & 53.73 & 54.55 & 56.67 & 52.54 \\
\hline \multirow{5}{*}{\multicolumn{2}{|c|}{ HRFA }} & DDX11 & c.G1819C & p.A607P & 9.38 & 5.66 & 14.15 & 10.16 \\
\hline & & DDX11 & c.C556T & p.R186W & 16.39 & 19.12 & 16.05 & 13.68 \\
\hline & & NBN & c.A511G & p.I171V & 50.43 & 56.96 & 48.81 & 46 \\
\hline & & PARP4 & c.G2695A & p.A899T & 48.89 & 33.66 & 39.71 & 48.91 \\
\hline & & SLX4 & c.C3812T & p.S1271F & 49.80 & 45.28 & 52.89 & 51.01 \\
\hline \multirow{3}{*}{\multicolumn{2}{|c|}{ NER }} & DCAF4 & c.C1034G & p.S345C & 45.88 & 45.38 & 40.56 & 41.95 \\
\hline & & & c.C478T & p.r160W & 99.22 & 85.29 & 58.24 & 59.83 \\
\hline & & $M C 1 R$ & c.G178T & p.V60L & 0 & 9.80 & 40.56 & 38.36 \\
\hline
\end{tabular}


To further verify the absence of genomic alterations after trabectedin and olaparib treatment, we performed copy number alteration analysis in sarcoma samples, comparing samples before and after treatment. No significant gene amplifications or copy loss, consistent with the loss of heterozygosity were found after treatment with trabectedin and olaparib in both cell lines and xenografts models compared to the parental counterpart (Figure 4).
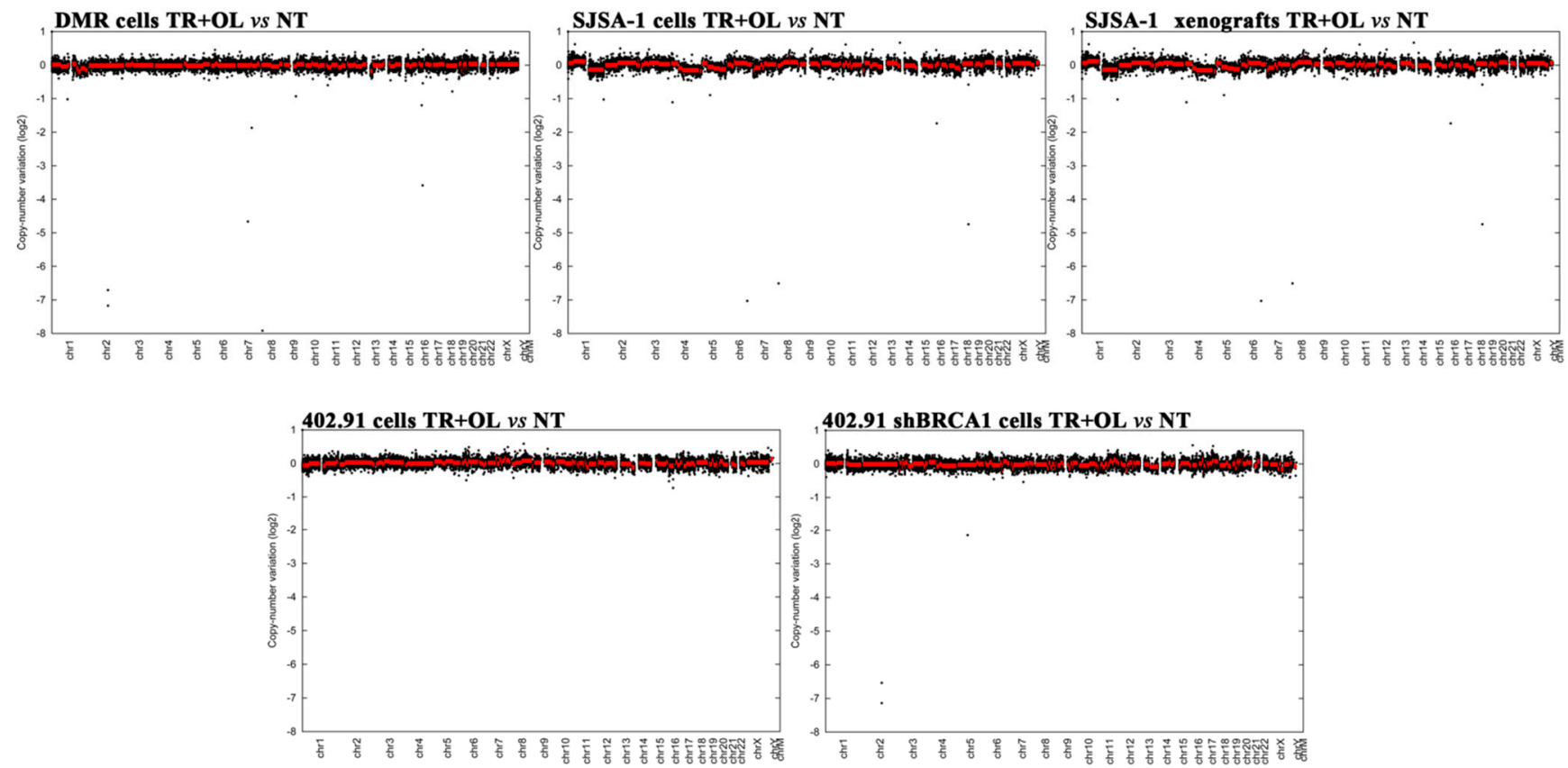

Figure 4. Copy number alterations calculated on WES data output after trabectedin + olaparib (TR + OL) treatment in DMR, SJSA-1, and SJSA-1 xenografts, 402.91, and 402.91 with stable silenced BRCA1 compared to relative untreated controls. Results are shown as Log2 variations in copy number (treated vs. untreated samples), plotted against chromosomal locations.

3.3. Treatment with Trabectedin and Olaparib Combination Did Not Influence Single-Base Substitution (SBS) Signatures

To mechanistically determine the impact of the PARP1 inhibitor olaparib and trabectedin combination on samples/cell lines, we performed mutational signature analysis, investigating each cell line and xenograft before and after olaparib and trabectedin combination. We also analyzed cell lines treated with the drug temozolomide as a positive control, because the signature SBS11 (in the COSMIC mutational signature reference) emerges after temozolomide treatment $[33,34]$. As shown in Figure 5, trabectedin and olaparib combination did not relevantly impact the mutational profiles of each cell line and xenograft treated with the olaparib and trabectedin combination. Conversely, as expected, temozolomide treatment induced a strong increment in Signature SBS11 in SJSA-1 T2 cells, showing a normalized score of 0.72 . Namely, this score is related to the increasing number of mutations and specifically, it showed that $72 \%$ of the mutations detected were predicted as induced by temozolomide. 

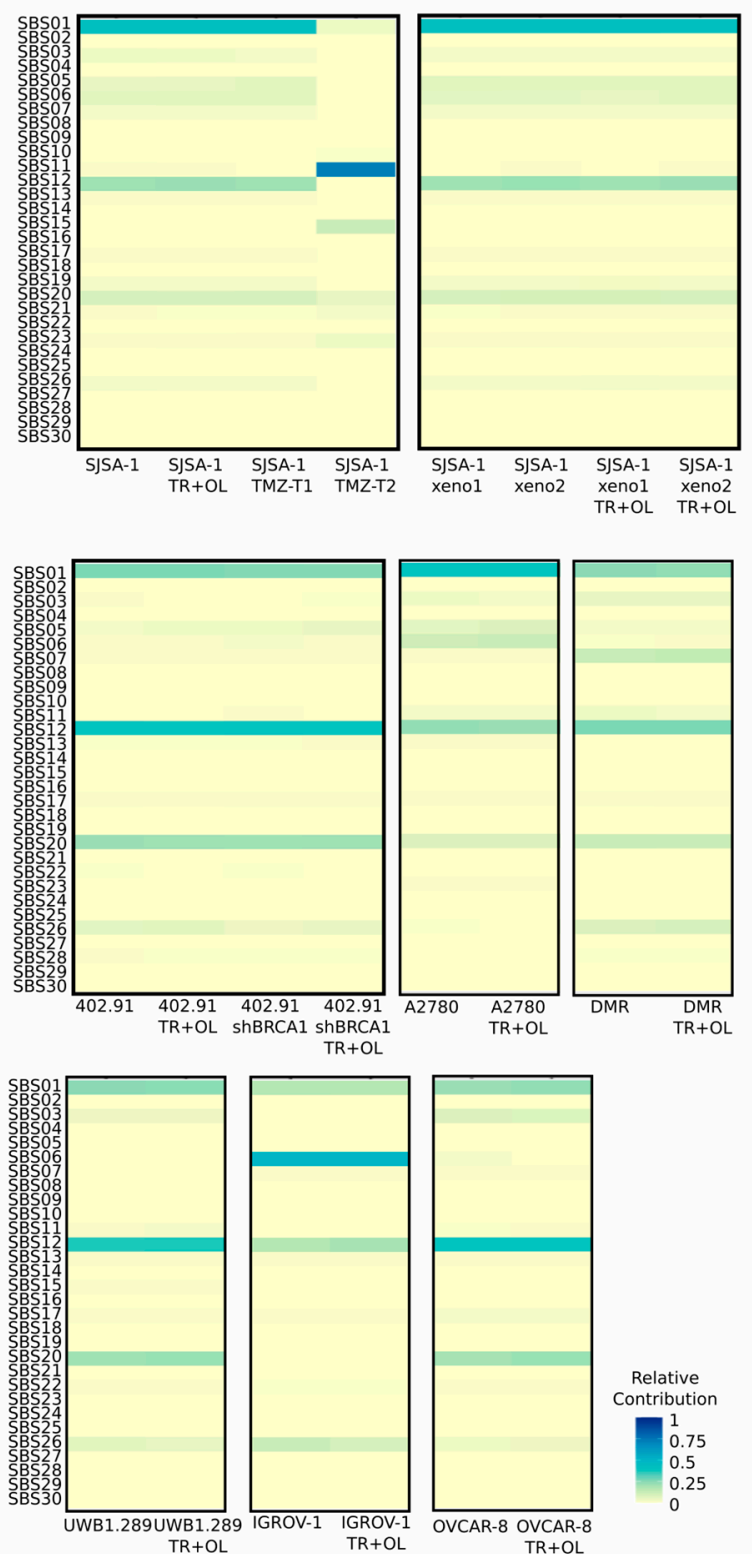

Figure 5. Mutational signatures analysis. Heatmap of normalized signature performed using COSMIC v2.0 as reference for the fitting analysis. Results are shown as the relative contribution of the specific SBS signature in treated and untreated samples for each specific cell line and treatment type. TR + OL: after three cycles of treatment with trabectedin + olaparib combination. TMZ: after temozolomide treatment.

\subsection{Trabectedin and Olaparib Treatment Did Not Impinge on Tumor Mutational Burden}

$\triangle \mathrm{TMB}$ was reported in cell lines and xenografts analyzed by WES or by the targeted Oncomine $\mathrm{TML}^{\mathrm{TM}}$ panel evaluating the somatic variations acquired after drug treatment. A very low $\triangle T M B$ was found after trabectedin and olaparib both in BRCA1/2-proficient and -defective cell lines, and in sarcoma xenografts, ruling out a mutagenic effect of the drug combination (Figure 6). Consistently, TMB analysis in tumor samples derived from patients treated with trabectedin and olaparib (five and six cycles, respectively), confirmed the absence of $\triangle \mathrm{TMB}$. As expected, SJSA-1 T2 cells (resistant to temozolomide) showed a massive increase in TMB, compared to baseline SJSA-1 T0 cells (Figure 6), confirming the result obtained by mutational signature analysis. 


\section{$\Delta$ (TMB) upon drug treatment}

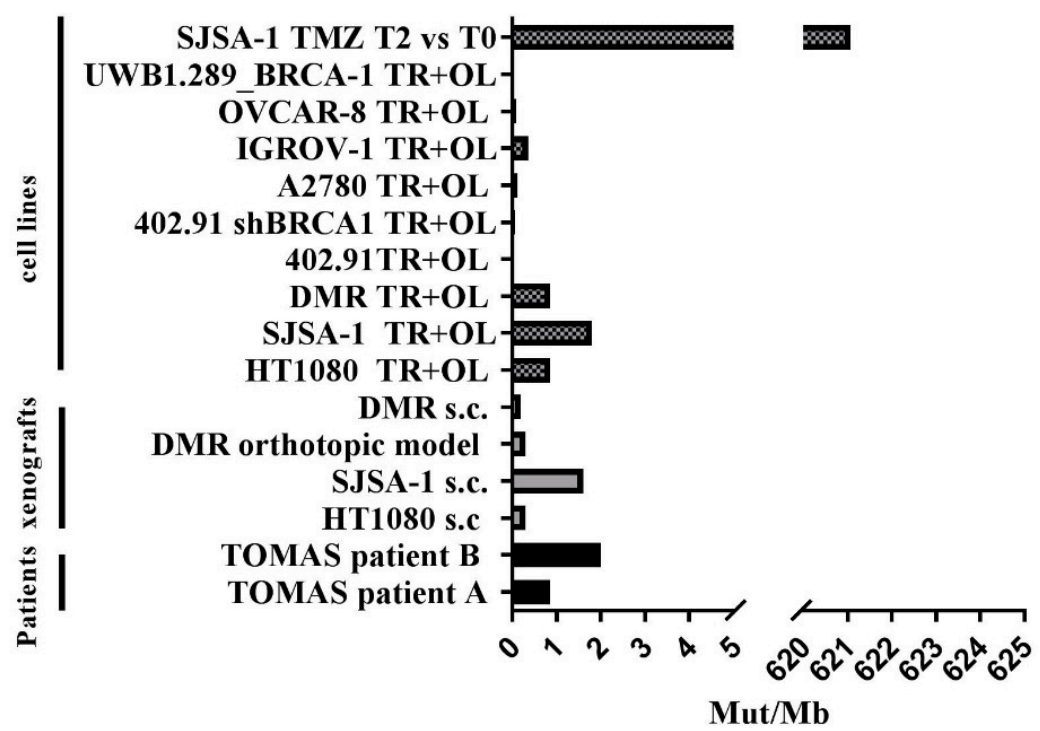

Figure 6. Variations in tumor mutational burden $(\triangle \mathrm{TMB})$ in a preclinical and translational platform of bone and soft tissue sarcomas treated with trabectedin + olaparib (TR + OL). TMB was calculated on somatic mutations identified on WES or targeted panel sequencing (Oncomine TML). Results are shown as alterations in the number of mutations per megabase, in treated samples vs. their untreated/baseline controls.

Notably, no clonality variation after trabectedin + olaparib combination was found, while in SJSA-1 cells treated with TMZ, it was possible to detect the appearance of a new clonal population due to TMZ treatment in SJSA-1 cells treated with TMZ (Figure 7).

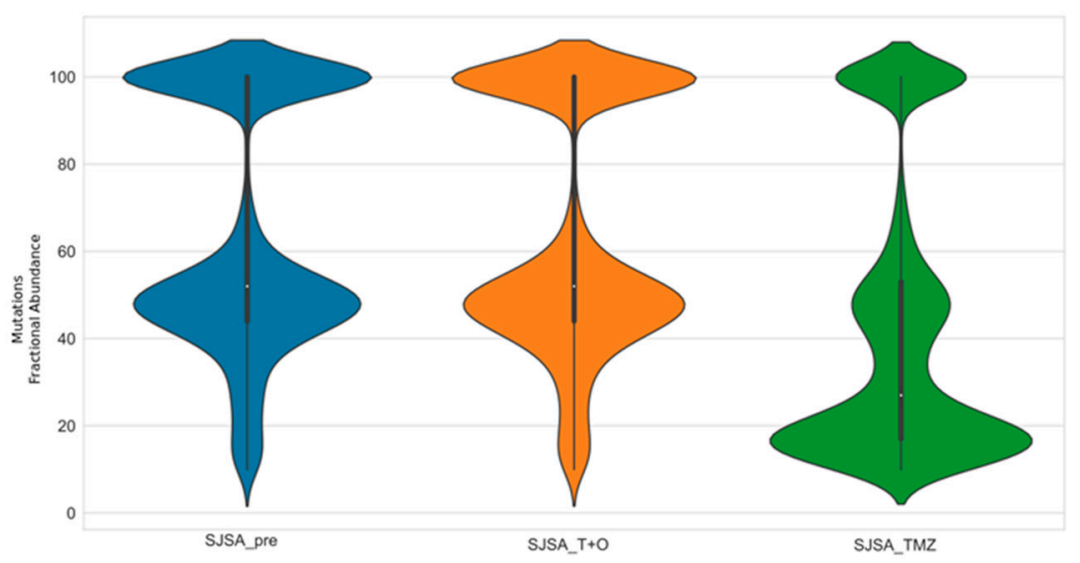

Figure 7. Clonality at baseline, after trabectedin + olaparib combination and after temozolomide treatment in the SJSA-1 cell line. The distribution of mutations fractional abundance for each treatment setting was showed SJSA_pre = SJSA-1 before treatment; SJSA_T + O = SJSA-1 after trabectedin + olaparib combination; SJSA_TMZ = SJSA-1 after temozolomide treatment.

\section{Discussion}

We explored the potential impact of an innovative therapeutic combination with trabectedin and olaparib, on the variation in TMB in advanced bone and soft tissue sarcomas, usually considered as "immune-cold tumors".

Indeed, compared to several epithelial tumors, bone and soft tissue sarcomas are not usually caused by mutagenic carcinogens known to introduce a high number of mutations 
in the human genome (such as tobacco smoke in lung cancer or UV light in melanoma) [37]. Furthermore, several mesenchymal tumors carry driver translocations (for instance, FUSCHOP $\mathrm{t}(12 ; 16)(\mathrm{q} 13 ; \mathrm{p} 11)$ in myxoid liposarcoma [38] or EWS/FLI1 $\mathrm{t}(11 ; 22)(\mathrm{q} 24 ; \mathrm{q} 12)$ and the likes in Ewing's sarcoma [39]) and are characterized by a relatively simple karyotype. A noteworthy exception is represented by gastrointestinal stromal tumors, wherein activating mutations in either KIT or PDGFR $\alpha$ genes drives tumor proliferation. In this context, trying to boost TMB by therapeutics in bone and soft tissue sarcomas could be a promising avenue to unleash response to immunotherapeutic strategies.

It should be emphasized that TMB is not a flawless predictive biomarker for immunotherapy response, but it is a useful proxy in terms of reproducibility, timing and large-scale feasibility of its evaluation [37]. In fact, not all nonsynonymous mutations will result in neoantigen generation, and not all novel peptides are effective immunogenic targets, adequately presented to the immune system [40].

By means of a robust preclinical platform, along with tumor samples of patients treated with this combination, we assessed both the mutational status of the DNA repair machinery and TMB before and after treatment with trabectedin and olaparib. We observed no change in mutational profiles, DDRR defects, copy number alterations and no relevant increase in TMB in either the preclinical or clinical setting. Overall, these data suggest that the combination of trabectedin and olaparib does not increase TMB, after three (preclinical) or five/six (patients) cycles of drug treatment. This information is reassuring regarding the feared risk of myelodysplasia/leukemia [19] reported with PARP1 inhibitors, but, at the same time, indicates that tumor-specific neoantigens are unlikely to be generated with this combination. Therefore, any future integration with ICI should be based on microenvironment modifications, rather than on TMB increase. This somewhat unexpected finding brings novel information to the foreground: chemotherapy and target therapy combination may end up in different genetic consequences, according to proficient or deficient pathways characterizing each specific tumor cell. Immune therapy with ICI represents a breakthrough in today's cancer treatment. However, in the sarcoma setting, only a minority of patients can really benefit from this intervention, at least in terms of overall survival and progression-free survival $[24,41-44]$. Thus, an extraordinary effort was made to both identify predictive key features in responsive tumors and modify the tumor/tumor microenvironment, aiming at improving patients' selection and enlarge the number of patients rationally eligible for ICI. Initially, PDL-1 expression was the first paradigmatic marker in candidate patients of immunotherapy. Later on, other biomarkers of response emerged, such as TMB, immune infiltrates and DDRR defects that showed a direct correlation with outcome after treatment with ICI $[1,5-7,37,45]$. These findings explained why sarcomas seldom respond to ICI and prompted studies to dissect sarcoma heterogeneity and attempts to modify the tumor and its microenvironment, making them more suitable for ICI. In this perspective, the combination of trabectedin and olaparib had a theoretically strong rationale: trabectedin and its derivatives modify the microenvironment, angiogenesis, and macrophage subsets $[46,47]$. The inhibition of PARP1, a key actor of DDRR, perpetuates this damage by impeding DNA repair [22]. In fact, we recently demonstrated that PARP1 inhibition enhances trabectedin-induced DNA damage and that treatment with this combination is feasible and active in advanced sarcoma patients [22,23]. In the present work, we explored whether tumor cells spared by this treatment might accumulate DNA damage and potentially be primed for subsequent immunotherapy with ICI. We assessed TMB, DDRR defects, gene copy number and mutational signatures in a preclinical and translational platform of bone and soft tissue sarcomas, treated with trabectedin and olaparib. At the end of these experiments, tumor cells spared by the combination both in preclinical and in patients' tumor samples did not show any modification in DDRR gene status, gene copy number or increase in TMB, nor did they relevantly impact on the mutational profiles of each cell line and xenograft treated with the olaparib and trabectedin combination. 
Given the fact that TMB measure is relative and that the number of clonal mutations is also relevant [48], we evaluated the clonality of our samples before and after treatment. We found no clonality variation in all samples treated with trabectedin + olaparib combination, while in our positive control (temozolomide treatment), the appearance of a new clonal population was observed. This is in line with our findings, highlighting the robustness of the selected control. Indeed, the increase in TMB could be linked to the intratumor heterogeneity increase (ITH). ITH is a known foe of molecular therapies because targeting a subpopulation of cancer cells (with a specific mutation) will lead to the Darwinian selective emergence of resistant clones [49]. In the immunotherapy field, this point is also under discussion. ITH appears as more a friend of immunotherapy than a foe, because it can be associated with the appearance of novel tumor antigens. In lung cancer [50] and melanoma [51], the data seem to be contradictory [52]: the high number of cancer neoantigens - created by strong subclonal expansions-attenuates anti-tumor immune responses to checkpoint blockade therapy. Recent data support these findings, suggesting that it is the percentage of tumor cells bearing each antigen within a tumor and not the absolute number of antigens that determines the efficacy of immune response [53]. In our study, we observed no differences either in clonal population distribution or in terms of ITH increase, after trabectedin + olaparib combination, differently from what we observed in the positive control (temozolomide treatment), in which novel clonal population emerged.

One intriguing aspect of our work was the observation that BRCA1 silencing in 402.91 cells (402.91shBRCA1) resulted in higher sensitivity to single agents, and consequent loss of evident synergistic effect. Indeed, it would be interesting to study the consequences of abrogated BRCA1 expression in in vivo models, treated with trabectedin + olaparib combination. This might reveal tumor microenvironment contributions to drug response and acquired genetic alterations in the presence or absence of BRCA1 loss.

In summary, on one hand, we confirmed that this treatment was safe and no hypermutation was identified in both studied models and patients. This finding is reassuring regarding PARP1i-related concerns about an increased risk of myelodysplasia/leukemia [19]. On the other hand, at least in terms of TMB, this combination does not seem an ideal induction therapy to ICI strategy. We acknowledge that other important aspects of crosstalk with the immune system might be evoked by this combination, such as modulation of the tumor microenvironment by both trabectedin and PARP1 inhibition [11,46,47] and activation of the stimulator of interferon genes (STING) pathway [54].

Other strategies to boost ICI response could also include multimodality treatment with chemotherapy and/or radiation therapy: both can increase TMB and, at the same time, improve local disease control. Furthermore, radiotherapy induces radiation-elicited T-cell activation ("radiation-induced viral mimicry", through STING pathway activation), and enhances cancer cell immunogenicity [55]. Genotoxic chemotherapy can generate similar effects, but not all chemotherapeutic agents have equal potency. For instance, the effect has been demonstrated with anthracyclines and platinum-derived chemotherapy [56]. Radiation therapy has been studied separately both in association with trabectedin in sarcomas, at the preclinical [57] and clinical [58] level, and with PARP1-inhibitors, which are known radiosensitizers [59]. Toxicity issues should always be considered in multimodality treatment, and moreover, the radiation dose needed to induce a significant TMB increase is still a matter of debate [60]. Indeed, conventional radiotherapy (e.g., 60 Gy in 2 Gyfractions) can introduce approximately one mutation per Megabase in the tumor genome, whilst techniques using larger doses per fraction (as stereotactic body radiotherapy) may obtain more relevant results [60].

We acknowledge that our work has limitations-in particular, the low number of available samples. Concerning the preclinical platform, it should be noted that establishing sarcoma PDXs is often challenging, also for the rarity of the disease. Regarding clinical, patient-derived samples, the only way to assess TMB variation was to collect samples from patients with very good responses, i.e., candidates for surgery after treatment. Otherwise, no post-treatment sample would have been available as the clinical study protocol did not 
envision post-progression biopsies. Only two samples were obtained, but given the fact that advanced/metastatic and pretreated sarcomas were enrolled, achieving two significant partial responses should already be considered as an exceptional occurrence. Exploring these findings in a larger patient cohort would be of interest to further confirm our results.

Finally, immuno-proficient preclinical models and a translational study on a larger cohort of treated patients will be instrumental for further investigations on these potentially actionable aspects. Moreover, a small subset of DDRR-defective tumors might be even more prone to both trabectedin + olaparib and ICI treatment, and molecular characterization of tumor samples could be a powerful tool for patient selection.

\section{Conclusions}

In conclusion, trabectedin and PARP1i treatment do not increase the TMB of sarcomas. Of course, it must be emphasized that sarcomas have more than eighty different histotypes and our conclusions may not apply to other rare histotypes. Other kinds of alkylating drugs, such as temozolomide, have been implicated in hypermutation, but the less safe toxicity profile may impinge on the clinical applicability of this drug, especially in combination with PARP1i [61]. Evaluating both genetic profiles and microenvironment changes in immunologically "cold" tumors, such as sarcomas, after conventional chemotherapy could be instrumental for developing novel insights to improve concomitant or sequence combination treatment with ICI.

Supplementary Materials: The following are available online at https: / www.mdpi.com/article / 10.3390/cancers13246295/s1. Table S1: Results of single nucleotide polymorfism identification (SNP_ID) for samples. Table S2: list of DDRR genes analyzed on WES data. Figure S1: Uncropped Western Blot Images.

Author Contributions: Y.P.: Conceptualization, methodology, validation, formal analysis, investigation, resources, data curation, writing —original draft preparation.; writing-review and editing; visualization; supervision; project administration; funding acquisition. G.C. (Giovanni Crisafulli): Conceptualization, methodology, software, validation, formal analysis, investigation, resources, data curation, writing - original draft preparation.; writing - review and editing; visualization; supervision. G.G. (Giorgia Giordano): Conceptualization, investigation, data curation, writing-review and editing. A.M.: Conceptualization, investigation, resources, data curation, writing-original draft preparation.; writing-review and editing; visualization. E.B.: Conceptualization, methodology, investigation; writing-review and editing. M.L.C.: investigation, data curation, writing-review and editing. G.C. (Giulia Chiabotto): Conceptualization, formal analysis, investigation, data curation, writing-original draft preparation.; writing—review and editing. S.B.: investigation, data curation, writing-review and editing. M.B.: methodology, investigation, data curation, writing-review and editing. E.M.: methodology, investigation, data curation, writing-review and editing. A.P.: methodology, investigation, data curation, writing - review and editing. V.L.: methodology, investigation, data curation, writing-review and editing. D.S.: Conceptualization, methodology, resources, writing-review and editing; visualization; supervision; funding acquisition, L.D.: Conceptualization, methodology, formal analysis, investigation, resources, data curation, writing-original draft preparation.; writing-review and editing; visualization. M.A.: Conceptualization, resources, writing-review and editing; visualization; supervision; project administration. B.K.: Conceptualization, writing-review and editing; visualization. A.B.: Conceptualization, methodology, resources, data curation, writing-original draft preparation.; writing-review and editing. G.G. (Giovanni Grignani): Conceptualization, resources, data curation, writing—original draft preparation.; writingreview and editing; visualization; supervision; project administration; funding acquisition. All authors have read and agreed to the published version of the manuscript.

Funding: This work was supported by Ministero della Salute-Ricerca Finalizzata, Giovani Ricercatori GR-2016-02362726 (Y.P.); Fondazione per la ricerca sui tumori dell'apparato muscoloscheletrico e rari ONLUS CRT RF = 2016-0917; RC 2021 Ministero della Salute (G.Gr.); AIRC IG 2019 - ID 23104 (G.Gr.); FPRC $5 \times 1000$ Ministero della Salute 2015 ImGen (G.Gr.); FPRC 5xmille MIUR 2014 (G.Gr.); AIRC 5 per Mille 2018 - ID. 21091 (A.B.); AIRC IG 2018 - ID. 21923 (A.B.); BiLiGeCT - Progetto PON ARS01_00492 (A.B.); FPRC $5 \times 1000$ Ministero della Salute 2015 ImGen (D.S.); AIRC IG 2017 - ID. 20259 (D.S.). 
Institutional Review Board Statement: The study was conducted according to the guidelines of the Declaration of Helsinki and approved by the Institutional Review Board and Ethics Committee of Candiolo Cancer Institute, FPO-IRCCS (N 202/2014). For animal studies: authorization Italian Ministry of Health (Prot. 21635.18, Aut.Min.834/2019-PR).

Informed Consent Statement: Informed consent was obtained from all subjects involved in the study.

Data Availability Statement: Data are available upon reasonable request.

Acknowledgments: Italian Sarcoma Group (ISG); the cell culture facility at Candiolo Cancer Institute (Raffaella Albano); the DNA sequencing facility at Candiolo Cancer Institute (Alice Bartolini). Radhika Srinivasan, for her careful English revision.

Conflicts of Interest: The funders had no role in the design of the study; in the collection, analysis, or interpretation of data; in the writing of the manuscript, or in the decision to publish the results. The authors declare no conflict of interest with regard to the present study. Potential COI outside of the present study: G.G. (Giovanni Grignani) has received fees for consulting and advisory board roles from PharmaMar, Lilly, Novartis, Bayer; and Eisai. A.M. has received travel expenses from PharmaMar. L.D. has received travel expenses from PharmaMar and Lilly. M.A. has received fees for consulting or advisory board roles from Bristol-Myers Squibb, Merck, and Roche. A.B. served in a consulting/advisory role for Illumina and Inivata, cofounder and shareholder of NeoPhore, and a member of the NeoPhore scientific advisory board. All other authors declare no competing interest.

\section{References}

1. Havel, J.; Chowell, D.; Chan, T.A. The Evolving Landscape of Biomarkers for Checkpoint Inhibitor Immunotherapy. Nat. Rev. Cancer 2019, 19, 133-150. [CrossRef] [PubMed]

2. Tumeh, P.C.; Harview, C.L.; Yearley, J.H.; Shintaku, I.P.; Emma, J.; Taylor, M.; Robert, L.; Chmielowski, B.; Spasic, M.; Henry, G.; et al. PD-1 Blockade Induces Responses by Inhibiting Adaptive Immune Resistance. Nature 2014, 515, 568-571. [CrossRef]

3. Fridman, W.H.; Pagès, F.; Sautès-Fridman, C.; Galon, J. The Immune Contexture in Human Tumours: Impact on Clinical Outcome. Nat. Rev. Cancer 2012, 12, 298-306. [CrossRef] [PubMed]

4. Riaz, N.; Havel, J.J.; Makarov, V.; Desrichard, A.; Sharfman, W.H.; Bhatia, S.; Hwu, W.; Gajewski, T.F.; Craig, L. Tumor and Microenvironment Evolution during Immunotherapy with Nivolumab. Cell 2017, 171, 934-949. [CrossRef]

5. Samstein, R.M.; Lee, C.H.; Shoushtari, A.N.; Hellmann, M.D. Tumor Mutational Load Predicts Survival after Immunotherapy across Multiple Cancer Types. Nat. Genet. 2019, 51, 202-206. [CrossRef]

6. Wu, Y.; Xu, J.; Du, C.; Wu, Y.; Xia, D.; Lv, W.; Hu, J. The Predictive Value of Tumor Mutation Burden on Efficacy of Immune Checkpoint Inhibitors in Cancers: A Systematic Review and Meta-Analysis. Front. Oncol. 2019, 9, 1161. [CrossRef] [PubMed]

7. Rizvi, N.A.; Hellmann, M.D.; Snyder, A.; Kvistborg, P.; Makarov, V.; Havel, J.J.; Lee, W.; Yuan, J.; Wong, P.; Ho, S.; et al. Mutational Landscape Determines Sensitivity to PD-1 Blockade in Non-Small Cell Lung Cancer. Science 2015, 348, 124-128. [CrossRef] [PubMed]

8. Snyder, A.; Makarov, V.; Merghoub, T.; Yuan, J. Genetic Basis for Clinical Response to CTLA-4 Blockade in Melanoma. N. Engl. J. Med. 2014, 371, 2189-2199. [CrossRef]

9. VanAllen, E.M.; Miao, D.; Schilling, B.; Shukla, S.A.; Blank, C.; Zimmer, L.; Sucker, A.; Hillen, U.; Geukes, M.H.; Goldinger, S.M.; et al. Genomic Correlates of Response to CTLA-4 Blockade in Metastatic Melanoma. Science 2015, 350, 207-211. [CrossRef]

10. Mcgranahan, N.; Furness, A.J.S.; Rosenthal, R.; Lyngaa, R.; Saini, S.K.; Jamal-hanjani, M.; Gareth, A.; Birkbak, N.J.; Hiley, C.T.; Watkins, T.B.K.; et al. Clonal Neoantigens Elicit T Cell Immunoreactivity and Sensitivity to Immune Checkpoint Blockade. Science 2016, 351, 1463-1469. [CrossRef]

11. Grignani, G.; Merlini, A.; Sangiolo, D.; Ambrosio, D.; Pignochino, Y. Delving into PARP Inhibition from Bench to Bedside and Back. Pharmacol. Ther. 2020, 206, 107446. [CrossRef] [PubMed]

12. Rodgers, K.; Mcvey, M. Error-Prone Repair of DNA Double-Strand Breaks. J. Cell Physiol. 2016, 231, 15-24. [CrossRef] [PubMed]

13. Howitt, B.E.; Shukla, S.A.; Sholl, L.M.; Ritterhouse, L.L.; Watkins, J.C.; Rodig, S.; Stover, E.; Strickland, K.C.; D'Andrea, A.D.; Wu, C.J.; et al. Association of Polymerase E-Mutated and Microsatellite-Instable Endometrial Cancers with Neoantigen Load, Number of Tumor-Infiltrating Lymphocytes, and Expression of PD-1 and PD-L1. JAMA Oncol. 2015, 1, 1319-1323. [CrossRef] [PubMed]

14. VanGool, I.C.; Eggink, F.A.; Freeman-mills, L.; Stelloo, E.; De Bruyn, M.; Palles, C.; Nout, R.A.; De Kroon, C.D.; Elisabeth, M.; Nijman, H.W.; et al. POLE Proofreading Mutations Elicit an Anti-Tumor Immune Response in Endometrial Cancer. Clin. Cancer Res. 2015, 21, 3347-3355. [CrossRef] [PubMed]

15. Strickland, K.C.; Howitt, B.E.; Shukla, S.A.; Rodig, S.; Ritterhouse, L.L.; Liu, J.F.; Garber, J.E.; Chowdhury, D.; Wu, C.J.; D'Andrea, A.D.; et al. Association and Prognostic Significance of BRCA1/2-Mutation Status with Neoantigen Load, Number of TumorInfiltrating Lymphocytes and Expression of PD-1/PD-L1 in High Grade Serous Ovarian Cancer. Oncotarget 2016, 7, 13587-13598. [CrossRef] [PubMed]

16. Martì, J.; Fernandez-Cortés, M.; Serrano-Sáenz, S.; Zamudio-martinez, E.; Delgado-bellido, D.; Garcia-diaz, A.; Oliver, F.J. The Multifactorial Role of PARP-1 in Tumor Microenvironment. Cancers 2020, 12, 739. [CrossRef] [PubMed] 
17. Vikas, P.; Borcherding, N.; Chennamadhavuni, A.; Garje, R. Therapeutic Potential of Combining PARP Inhibitor and Immunotherapy in Solid Tumors. Front. Oncol. 2020, 10, 570. [CrossRef]

18. Poveda, A.; Floquet, A.; Ledermann, J.A.; Asher, R.; Penson, R.T.; Oza, A.M.; Korach, J.; Huzarski, T.; Pignata, S.; Friedlander, M.; et al. Olaparib Tablets as Maintenance Therapy in Patients with Platinum-Sensitive Relapsed Ovarian Cancer and a BRCA1/2 Mutation (SOLO2/ENGOT-Ov21): A Final Analysis of a Double-Blind, Randomised, Placebo-Controlled, Phase 3 Trial. Lancet Oncol. 2021, 22, 620-631. [CrossRef]

19. Morice, P.; Leary, A.; Dolladille, C.; Chrétien, B.; Poulain, L.; González-martín, A.; Moore, K.; Reilly, E.M.O.; Ray-coquard, I.; Alexandre, J. Myelodysplastic Syndrome and Acute Myeloid Leukaemia in Patients Treated with PARP Inhibitors: A Safety Meta-Analysis of Randomised Controlled Trials and a Retrospective Study of the WHO Pharmacovigilance Database. Lancet Haematol. 2021, 8, 122-134. [CrossRef]

20. Ordóñez, J.L.; Amaral, A.T.; Carcaboso, A.M.; Herrero-Martín, D.; Del Carmen García-Macías, M.; Sevillano, V.; Alonso, D.; Pascual-Pasto, G.; San-Segundo, L.; Vila-Ubach, M.; et al. The PARP Inhibitor Olaparib Enhances the Sensitivity of Ewing Sarcoma to Trabectedin. Oncotarget 2015, 6, 18875-18890. [CrossRef] [PubMed]

21. Laroche, A.; Chaire, V.; Le Loarer, F.; Algéo, M.P.; Rey, C.; Tran, K.; Lucchesi, C.; Italiano, A. Activity of Trabectedin and the PARP Inhibitor Rucaparib in Soft-Tissue Sarcomas. J. Hematol. Oncol. 2017, 10, 84. [CrossRef]

22. Pignochino, Y.; Capozzi, F.; D’Ambrosio, L.; Dell'Aglio, C.; Basiricò, M.; Canta, M.; Lorenzato, A.; Vignolo Lutati, F.; Aliberti, S.; Palesandro, E.; et al. PARP1 Expression Drives the Synergistic Antitumor Activity of Trabectedin and PARP1 Inhibitors in Sarcoma Preclinical Models. Mol. Cancer 2017, 16, 86. [CrossRef] [PubMed]

23. Grignani, G.; Ambrosio, L.D.; Pignochino, Y.; Palmerini, E.; Zucchetti, M.; Boccone, P.; Aliberti, S.; Paolo, A.; Tos, D.; Novara, L.; et al. Trabectedin and Olaparib in Patients with Advanced and Non-Resectable Bone and Soft-Tissue Sarcomas (TOMAS): An Open-Label, Phase 1b Study from the Italian Sarcoma Group. Lancet Oncol. 2018, 19, 1360-1371. [CrossRef]

24. Petitprez, F.; de Reyniès, A.; Keung, E.Z.; Chen, T.W.W.; Sun, C.M.; Calderaro, J.; Jeng, Y.M.; Hsiao, L.P.; Lacroix, L.; Bougoüin, A.; et al. B Cells Are Associated with Survival and Immunotherapy Response in Sarcoma. Nature 2020, 577, 556-560. [CrossRef] [PubMed]

25. Corti, G.; Bartolini, A.; Crisafulli, G.; Novara, L.; Rospo, G.; Montone, M.; Negrino, C.; Mussolin, B.; Buscarino, M.; Isella, C.; et al. A Genomic Analysis Workflow for Colorectal Cancer Precision Oncology. Clin. Colorectal. Cancer 2019, 18, 91-101. [CrossRef]

26. Crisafulli, G.; Mussolin, B.; Cassingena, A.; Montone, M.; Bartolini, A.; Barault, L.; Martinetti, A.; Morano, F.; Pietrantonio, F.; Sartore-Bianchi, A.; et al. Whole Exome Sequencing Analysis of Urine Trans-Renal Tumour DNA in Metastatic Colorectal Cancer Patients. ESMO Open 2019, 4, e000572. [CrossRef]

27. Conway, T.; Wazny, J.; Bromage, A.; Tymms, M.; Williams, E.D.; Beresford-Smith, B. Xenome-A Tool for Classifying Reads from Xenograft Samples. Bioinformatics 2012, 28, 172-178. [CrossRef] [PubMed]

28. Svensson, F.; Lang, T.; Johansson, M.E.V.; Hansson, G.C. The Central Exons of the Human MUC2 and MUC6 Mucins Are Highly Repetitive and Variable in Sequence between Individuals. Sci. Rep. 2018, 8, 17503. [CrossRef]

29. Russo, M.; Crisafulli, G.; Sogari, A.; Reilly, N.M.; Arena, S.; Bartolini, A.; Amodio, V.; Magrì, A.; Novara, L.; Sarotto, I.; et al. Adaptive Mutability of Colorectal Cancers in Response to Targeted Therapies. Science 2019, 366, 1473-1480. [CrossRef]

30. Siravegna, G.; Lazzari, L.; Crisafulli, G.; Sartore-Bianchi, A.; Mussolin, B.; Cassingena, A.; Martino, C.; Lanman, R.B.; Nagy, R.J.; Fairclough, S.; et al. Radiologic and Genomic Evolution of Individual Metastases during HER2 Blockade in Colorectal Article Radiologic and Genomic Evolution of Individual Metastases during HER2 Blockade in Colorectal Cancer. Cancer Cell 2018, 34, 148-162. [CrossRef]

31. Germano, G.; Lamba, S.; Rospo, G.; Barault, L.; Magrì, A.; Maione, F.; Russo, M.; Crisafulli, G.; Bartolini, A.; Lerda, G.; et al. Inactivation of DNA Repair Triggers Neoantigen Generation and Impairs Tumour Growth. Nature 2017, 552, 116-120. [CrossRef]

32. Bergstrom, E.N.; Huang, M.N.; Mahto, U.; Barnes, M.; Stratton, M.R.; Rozen, S.G.; Alexandrov, L.B. SigProfilerMatrixGenerator: A Tool for Visualizing and Exploring Patterns of Small Mutational Events. BMC Genom. 2019, 20, 685. [CrossRef]

33. Alexandrov, L.B.; Nik-Zainal, S.; Wedge, D.C.; Aparicio, S.A.J.R.; Behjati, S.; Biankin, A.V.; Bignell, G.R.; Bolli, N.; Borg, A.; Børresen-Dale, A.; et al. Signatures of Mutational Processes in Human Cancer. Nature 2013, 500, 415-421. [CrossRef] [PubMed]

34. Alexandrov, L.B.; Kim, J.; Haradhvala, N.J.; Huang, M.N.; Ng, A.W.T.; We, Y.; Boot, A.; Covington, K.R.; Gordenin, D.A.; Bergstrom, E.N.; et al. The Repertoire of Mutational Signatures in Human Cancer. Nature 2020, 578, 94-101. [CrossRef] [PubMed]

35. Díaz-Gay, M.; Vila-Casadesús, M.; Franch-Expósito, S.; Hernández-Illán, E.; Lozano, J.J.; Castellví-Bel, S. Mutational Signatures in Cancer (MuSiCa): A Web Application to Implement Mutational Signatures Analysis in Cancer Samples. BMC Bioinform. 2018, 19, 224. [CrossRef]

36. Blokzijl, F.; Janssen, R.; Van Boxtel, R.; Cuppen, E. MutationalPatterns: Comprehensive Genome-Wide Analysis of Mutational Processes. Genome Med. 2018, 10, 33. [CrossRef]

37. Chan, T.A.; Yarchoan, M.; Jaffee, E.; Swanton, C.; Quezada, S.A.; Stenzinger, A.; Peters, S. Development of tumor mutation burden as an immunotherapy biomarker: Utility for the oncology clinic. Ann. Oncol. 2019, 30, 44-56. [CrossRef] [PubMed]

38. Knight, J.C.; Renwick, P.J.; Dal Cin, P.; Van den Berghe, H.; Fletcher, C.D. Translocation t(12;16)(q13;p11) in myxoid liposarcoma and round cell liposarcoma: Molecular and cytogenetic analysis. Cancer Res. 1995, 55, 24-27. [PubMed]

39. Turc-Carel, C.; Aurias, A.; Mugneret, F.; Lizard, S.; Sidaner, I.; Volk, C.; Thiery, J.P.; Olschwang, S.; Philip, I.; Berger, M.P. Chromosomes in Ewing's sarcoma. I. An evaluation of 85 cases of remarkable consistency of t(11;22)(q24;q12). Cancer Genet. Cytogenet. 1988, 32, 229-238. [CrossRef] 
40. Snyder, A.; Chan, T.A. Immunogenic peptide discovery in cancer genomes. Curr. Opin Genet. Dev. 2015, 30, 7-16. [CrossRef] [PubMed]

41. Italiano, A.; Bessede, A.; Bompas, E.; Piperno-Neumann, S.; Chevreau, C.; Penel, N.; Bertucci, F.; Toulmonde, M.; Bellera, C.A.; Guegan, J.; et al. PD1 Inhibition in Soft-Tissue Sarcomas with Tertiary Lymphoid Structures: A Multicenter Phase II Trial. J. Clin. Oncol. 2021, 39 (Suppl. 15), 11507. [CrossRef]

42. Toulmonde, M.; Brahmi, M.; Giraud, A.; Bessede, A.; Kind, M.; Toulza, E.; Pulido, M.; Cousin, S.; Bourcier, K.; Albert, S.; et al TRAMUNE, a Phase Ib Study Combining Trabectedin and Durvalumab, Results of the Expansion Cohort in Patients with Advanced Pretreated Soft Tissue Sarcomas. Ann. Oncol. 2020, 31, S1199. [CrossRef]

43. Blay, J.Y.; Penel, N.; Ray-Coquard, I.L.; Cousin, S.; Bertucci, F.; Bompas, E.; Eymard, J.C.; Saada-Bouzid, E.; Soulie, P.; BoudouRouquette, P.; et al. High Clinical Activity of Pembrolizumab in Chordoma, Alveolar Soft Part Sarcoma (ASPS) and Other Rare Sarcoma Histotypes: The French AcSé Pembrolizumab Study from Unicancer. J. Clin. Oncol. 2021, 39, 11520. [CrossRef]

44. Hindi, N.; Rosenbaum, E.; Jonczak, E.; Hamacher, R.; Rutkowski, P.; Skryd, A.; Connolly, E.; Blay, J.Y.; Gutierrez, A.; Bogefors, C.; et al. Retrospective World-Wide Registry on the Efficacy of Immune Checkpoint Inhibitors in Alveolar Soft Part Sarcoma: Updated Results from Sixty Patients. J. Clin. Oncol. 2021, 39, 11564. [CrossRef]

45. Davis, A.A.; Patel, V.G. The Role of PD-L1 Expression as a Predictive Biomarker: An Analysis of All US Food and Drug Administration (FDA) Approvals of Immune Checkpoint Inhibitors. J. Immunother. Cancer 2019, 7, 278. [CrossRef] [PubMed]

46. Germano, G.; Frapolli, R.; Simone, M.; Tavecchio, M.; Erba, E.; Pesce, S.; Pasqualini, F.; Grosso, F.; Sanfilippo, R.; Casali, P.G.; et al. Antitumor and Anti-Inflammatory Effects of Trabectedin on Human Myxoid Liposarcoma Cells. Cancer Res. 2010, 70, $2235-2244$. [CrossRef]

47. Germano, G.; Frapolli, R.; Belgiovine, C.; Anselmo, A.; Pesce, S.; Liguori, M.; Erba, E.; Uboldi, S.; Zucchetti, M.; Pasqualini, F.; et al. Role of Macrophage Targeting in the Antitumor Activity of Trabectedin. Cancer Cell 2013, 23, 249-262. [CrossRef] [PubMed]

48. Turajlic, S.; Sottoriva, A.; Graham, T.; Swanton, C. Resolving genetic heterogeneity in cancer. Nat. Rev. Genet. 2019, 20, 404-416. [CrossRef]

49. Siravegna, G.; Mussolin, B.; Buscarino, M.; Corti, G.; Cassingena, A.; Crisafulli, G.; Ponzetti, A.; Cremolini, C.; Amatu, A.; Lauricella, C.; et al. Clonal evolution and resistance to EGFR blockade in the blood of colorectal cancer patients. Nat. Med. 2015, 21, 827. [CrossRef]

50. Rosenthal, R.; Cadieux, E.L.; Salgado, R.; Bakir, M.A.; Moore, D.A.; Hiley, C.T.; Lund, T.; Tanić, M.; Reading, J.L.; Joshi, K.; et al. Neoantigen-directed immune escape in lung cancer evolution. Nature 2019, 567, 479-485. [CrossRef]

51. Wolf, Y.; Bartok, O.; Patkar, S.; Eli, G.B.; Cohen, S.; Litchfield, K.; Levy, R.; Jiménez-Sánchez, A.; Trabish, S.; Lee, J.S.; et al. UVB-Induced Tumor Heterogeneity Diminishes Immune Response in Melanoma. Cell 2019, 179, 219-235. [CrossRef] [PubMed]

52. McGranahan, N.; Swanton, C. Neoantigen quality, not quantity. Sci. Transl. Med. 2019, 11, eaax7918. [CrossRef] [PubMed]

53. Gejman, R.S.; Chang, A.Y.; Jones, H.F.; DiKun, K.; Hakimi, A.A.; Schietinger, A.; Scheinberg, D.A. Rejection of immunogenic tumor clones is limited by clonal fraction. Elife 2018, 7, e41090. [CrossRef] [PubMed]

54. Ding, L.; Kim, H.J.; Wang, Q.; Kearns, M.; Jiang, T.; Ohlson, C.E.; Li, B.B.; Xie, S.; Liu, J.F.; Stover, E.H.; et al. PARP Inhibition Elicits STING-Dependent Antitumor Immunity in Brca1-Deficient Ovarian Cancer. Cell Rep. 2018, 25, 2972-2980. [CrossRef]

55. Lhuillier, C.; Rudqvist, N.P.; Elemento, O.; Formenti, S.C.; Demaria, S. Radiation therapy and anti-tumor immunity: Exposing immunogenic mutations to the immune system. Genome Med. 2019, 11, 40. [CrossRef]

56. Kroemer, G.; Galluzzi, L.; Kepp, O.; Zitvogel, L. Immunogenic cell death in cancer therapy. Annu Rev. Immunol 2013, 31, 51-72. [CrossRef] [PubMed]

57. Romero, J.; Zapata, I.; Córdoba, S.; Jimeno, J.M.; López-Martín, J.A.; Tercero, J.C.; De La Torre, A.; Vargas, J.A.; Molerón, R.; Sánchez-Prieto, R. In vitro radiosensitisation by trabectedin in human cancer cell lines. Eur. J. Cancer 2008, 44, 1726-1733. [CrossRef]

58. Martin-Broto, J.; Hindi, N.; Lopez-Pousa, A.; Peinado-Serrano, J.; Alvarez, R.; Alvarez-Gonzalez, A.; Italiano, A.; Sargos, P.; Cruz-Jurado, J.; Isern-Verdum, J.; et al. Assessment of Safety and Efficacy of Combined Trabectedin and Low-Dose Radiotherapy for Patients with Metastatic Soft-Tissue Sarcomas: A Nonrandomized Phase 1/2 Clinical Trial. JAMA Oncol. 2020, 6, 535-541. [CrossRef]

59. Jannetti, S.A.; Zeglis, B.M.; Zalutsky, M.R.; Reiner, T. Poly(ADP-Ribose)Polymerase (PARP) Inhibitors and Radiation Therapy. Front. Pharmacol. 2020, 11, 170. [CrossRef]

60. Giordano, F.A.; Veldwijk, M.R.; Herskind, C.; Wenz, F. Radiotherapy, tumor mutational burden, and immune checkpoint inhibitors: Time to do the math. Strahlenther. Onkol. 2018, 194, 873-875. [CrossRef]

61. Plummer, R.; Jones, C.; Middleton, M.; Wilson, R.; Evans, J.; Olsen, A.; Curtin, N.; Boddy, A.; Mchugh, P.; Newell, D.; et al. Phase I Study of The Poly (ADP-Ribose) Polymerase Inhibitor, AG014699, In Combination with Temozolomide in Patients with Advanced Solid Tumors. Clin. Cancer Res. 2008, 14, 7917-7923. [CrossRef] [PubMed] 